NISTIR 7910

\title{
Alternative Methods of Latent Fingerprint Enhancement and Metrics for Comparing Them
}

\author{
Alfred S. Carasso
}

http://dx.doi.org/10.6028/NIST.IR.7910

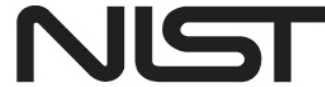

National Institute of Standards and Technology U.S. Department of Commerce 


\title{
Alternative Methods of Latent Fingerprint Enhancement and Metrics for Comparing Them
}

\author{
Alfred S. Carasso \\ Applied and Computational Mathematics Division \\ Information Technology Laboratory
}

http://dx.doi.org/10.6028/NIST.IR.7910

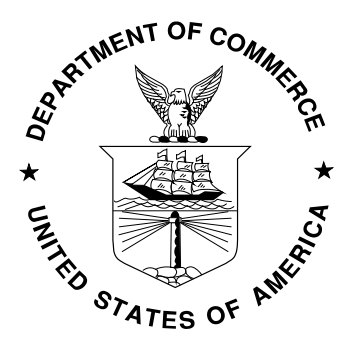

U.S. Department of Commerce Rebecca Blank, Acting Secretary

National Institute of Standards and Technology Patrick D. Gallagher, Under Secretary of Commerce for Standards and Technology and Director 


\title{
ALTERNATIVE METHODS OF LATENT FINGERPRINT ENHANCEMENT AND METRICS FOR COMPARING THEM
}

\author{
ALFRED S. CARASSO*
}

\begin{abstract}
Digital enhancement of latent fingerprints using Photoshop processing ${ }^{1}$ is the preferred methodology among law enforcement forensic experts. This report explores alternative enhancement methods that are independent of Photoshop, relying instead on such widely used scientific image analysis packages as MATLAB, IDL, and PV-WAVE. In addition, standard image metrics are applied to the processed images and shown capable of distinguishing among various types of enhancements. The discussion is anchored around three illustrative examples, consisting of 8 bit greyscale latent fingerprint image pairs extracted from the NIST forensic database. It is shown that alternative enhancement methods exist that can recover potentially significant fine scale information, such as may elude standard forensic Photoshop processing.
\end{abstract}

Key words. latent fingerprints; digital image enhancement; image gradient norms; total variation norm.

1. Introduction. This report is a contribution to the FY 2012 research project Metrics for Manipulation and Enhancement of Forensic Images, sponsored by the NIST Information Technology Laboratory. That project is primarily focused on the processes used for digital image enhancement of poor quality latent fingerprints left unintentionally at a crime scene. To facilitate this study, law enforcement forensic experts provided NIST with a database of fingerprint image pairs, before and after enhancement. Such enhancements result exclusively from Adobe Photoshop image processing, which is the methodology preferred by law enforcement professionals.

The discussion in this report is anchored around three illustrative examples, labeled (A), (B), and (C), and consisting of 8 bit greyscale latent fingerprint image pairs, extracted from the Originals/White Powder Developed Prints/NIST Study series in the above database.

In recent years, skepticism has occasionally been expressed about the reliability of digital enhancements of latent fingerprints, [1], [2]. The Photoshop methodology does not provide a digital record of the various steps applied in enhancing the image. Moreover, the particular combination of Photoshop steps used in the process may inadvertently eliminate vital fine scale information, information that might $e x-$ clude a suspect. This report discusses alternative approaches to latent fingerprint enhancement that are independent of Photoshop. Consideration of such alternative enhancements, alongside the traditional Photoshop result, may better inform the subsequent, crucial, matching and identification procedure. An important characteristic of the proposed methods is that they yield reproducible results, as they are based on the use of simple commands from such widely used scientific image analysis software packages as MATLAB [3], IDL [4], and PV-WAVE [5]. The uses and purposes of these commands are documented and illustrated in the accompanying user's manuals. In addition, [6] provides a thorough discussion of the theoretical ideas underlying the various enhancement techniques available in the above packages. Note that implementations vary among different packages, and the same enhancement technique can

*Applied and Computational Mathematics Division, National Institute of Standards and Technology, Gaithersburg, MD 20899. (alfred.carasso@nist.gov).

${ }^{1}$ Mention of commmercial products or services in this report does not imply NIST appproval or endorsement of these products or services, nor does it imply that such products or services are necessarily the best available for the intended purpose. 
produce slightly different images in different packages. Such enhancement methods are designed to bring into better visual range potentially significant structures that are present in the original image, but not easily discernible.

This report includes software routines that can be used with MATLAB and IDL packages to enhance latent fingerprints. Here, these routines are applied to the three original latent print images $(\mathrm{A}),(\mathrm{B})$, and $(\mathrm{C})$. It should be noted that image $(\mathrm{C})$ is substantially different from image (A), although both images look similar at first glance. Each of these images is subjected to five distinct alternative enhancements, in addition to the Photoshop enhancement included in the NIST database. Standard image norms are then calculated for each processed image. Such norms confirm and quantify the noticeable differences that are evident in some of these enhancements.

1.1. Default parameter values and further possible enhancements. In the examples discussed below using the software routines in Section 3, only the simplest forms of image analysis commands are used. In fact, each command can be applied with a variety of options by specifying desired values in an associated parameter list. Here, such parameter values are omitted, resulting in default values being assumed. Using the routines of Section 3 with more sophisticated parameter choices, can produce a much larger variety of distinct enhancements than are shown below for images (A), (B) and (C).

2. Image metrics. The latent fingerprint images discussed in this report are 8 bit grey scale tiff images, with intensity values ranging from 0 to 255 . These images are large, rectangular, and of diverse sizes, with the $y$ dimension exceeding the $x$ dimension. The software routines described in Section 3 can accept and process these diverse size images. However, post processing, all images in this report are reformatted as follows in order to simplify image manipulations, and enable viewing of multiple post-processed images on a single screen. The $y$ size of each image is reduced to 1024 pixels; the $x$ size is then reduced by the appropriate amount so as to maintain the original $x / y$ ratio. Next, the $x$ size of the image is increased to 1024 pixels by zero padding in the $x$ direction. All image norms defined below and used in Tables $1-6$, refer to these zero padded $1024 \times 1024$ pixel images, denoted by $f(x, y)$.

The following discrete $L^{1}$ and $L^{2}$ image norms, as well as the discrete $L^{1}$ and $L^{2}$ image gradient norms, will be found helpful in distinguishing among the various enhancement approaches discussed below. The image gradient norms are particularly sensitive to the presence of fine scale information in the processsed image.

$$
\|f\|_{p}=\left\{(1024)^{-2} \sum_{x, y=1}^{1024}|f(x, y)|^{p}\right\}^{1 / p}, \quad p=1,2,
$$

and

$$
\|\nabla f\|_{p}=\left\{(1024)^{-2} \sum_{x, y=1}^{1023}\left(\left\{f^{x}(x, y)\right\}^{2}+\left\{f^{y}(x, y)\right\}^{2}\right)^{p / 2}\right\}^{1 / p}, \quad p=1,2,
$$

where

$$
\begin{aligned}
& f^{x}(x, y)=(1024)(f(x+1, y)-f(x, y)), \\
& f^{y}(x, y)=(1024)(f(x, y+1)-f(x, y)) .
\end{aligned}
$$


The norm $\|\nabla f\|_{1}$ in Eq.(2), sometimes called the discrete 'Total Variation' or $T V$ norm, plays an important role in image analysis. Unexpectedly, the $L^{2}$ image gradient norm, $\|\nabla f\|_{2}$, turns out to be more significant in the examples below.

3. Alternative enhancements using simple software routines. The enhancement methods used in this report are itemized below. The sketchy descriptions in this itemized list can be supplemented by consulting [3], [4], and [5]. In particular, the various options available with each method are discussed in detail in these user's guides. Instructive analytical discussions concerning these and other enhancement techniques may be found in [6].

- Contrast Adjustment. (MATLAB imadjust; IDL bytscl). This method enhances the image by changing underlying image intensities through stretching and brightening the original intensity range.

- Sobel Filtering. (IDL sobel). This is an edge enhancement technique based on differentiating the image, using a special difference approximation to the gradient at each point.

- Unsharp Masking. (IDL unsharp_mask). This is a useful method for highpass filtering, that highlights edges and small sharp features in the displayed image. It consists of subtracting a Gaussian smoothed version from the original image, so as to better emphasize the high frequency components in the original.

- Histogram Equalization. (MATLAB histeq; IDL hist_equal). In many images of interest, pixel values tend to reside in a few narrow subintervals of the full 8 bit interval $[0,255]$. One can maximize the information content in the displayed image by spreading the distribution of pixel values, so as to more evenly cover the full range from 0 to 255 . This helps bring small scale details into better visual range, while typically changing the appearance of the image.

- Adaptive Histogram Equalization. (IDL adapt_hist_equal). This is a more refined form of histogram equalization, whereby contrast enhancement is adapted to the local region surrounding each pixel. This method is believed to produce superior images.

3.1. MATLAB routine. The following MATLAB m-file is used for contrast adjustment and histogram equalization. Additional commands can be added to this $\mathrm{m}$-file, and more sophisticated versions of each command can be created by specifying appropriate parameters.

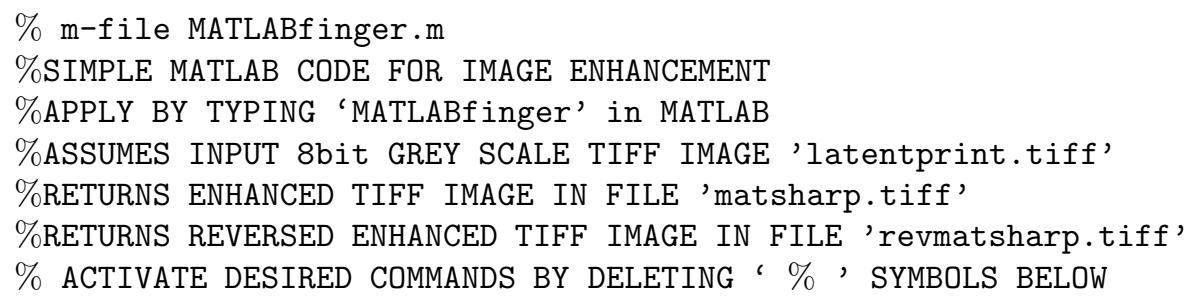




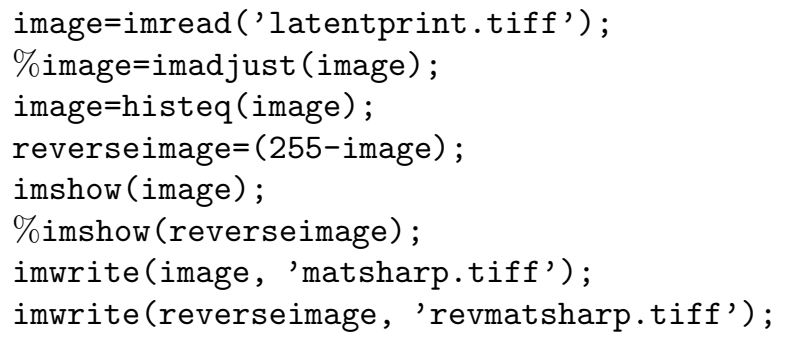

3.2. IDL routine. This routine is used for Sobel filtering, unsharp masking, and adaptive histogram equalization. As above, additional commands can be added, and more sophisticated versions of each of these commands can be created.

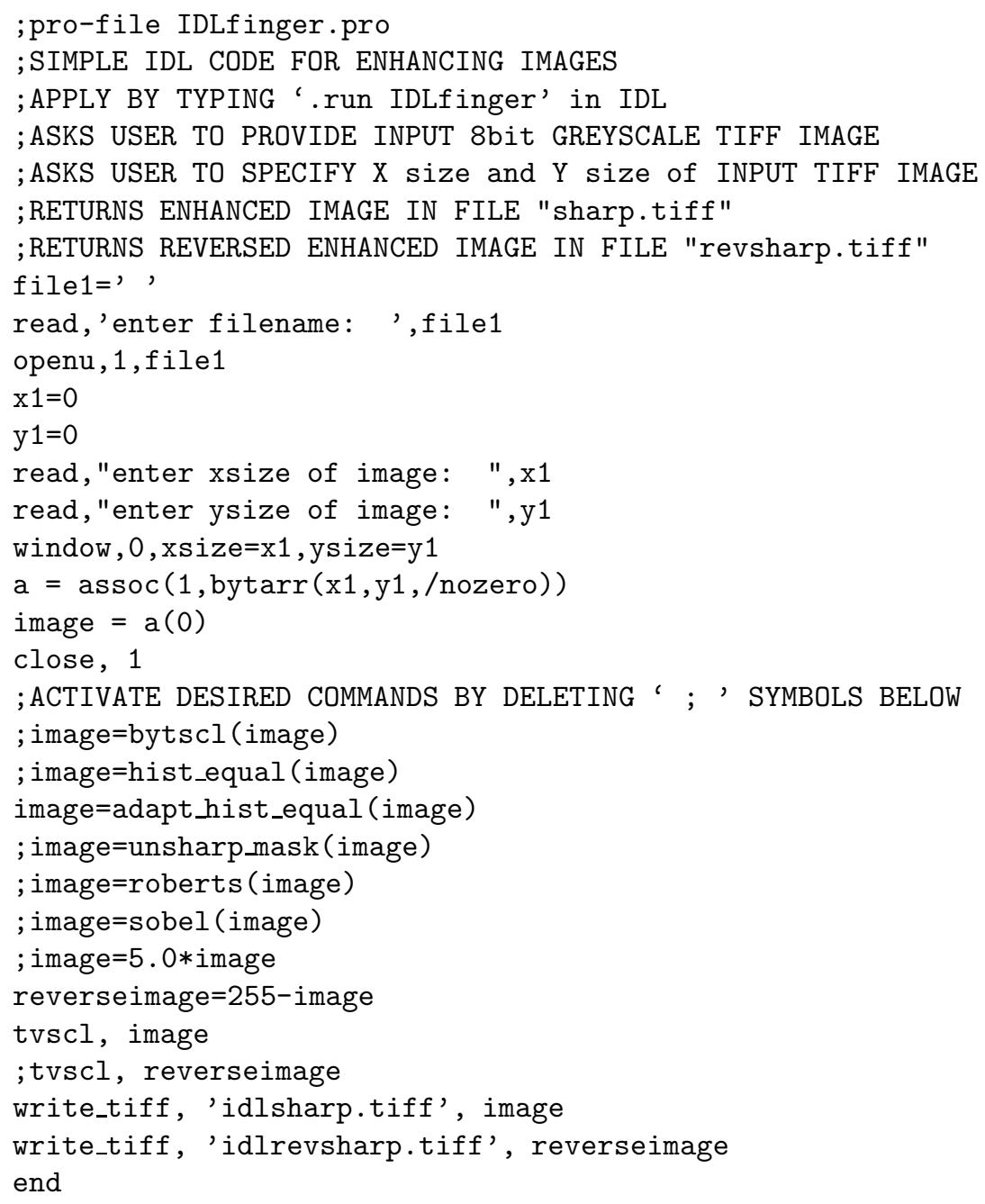

4. Enhancing images (A), (B), and (C). Figure 1 shows the results of applying the MATLAB commands for contrast adjustment and histogram equalization to the original latent print (A), and compares them to the forensic Photoshop process. 
The corresponding reverse images are shown in Figure 2 on the adjoining page. The MATLAB contrast adjusted image in Figure 1 differs somewhat from the Photoshop image, a difference that is picked up in the $L^{2}$ image and image gradient norms in Table 1, although not in the corresponding $L^{1}$ norms. Also, the reverse MATLAB contrast image is sharper than the reverse Photoshop image. However, the MATLAB histogram equalized image in Figure 1, and its reverse counterpart in Figure 2, are vastly different enhancements. This is reflected in the substantially larger $L^{1}$ and $L^{2}$ image and image gradient norms in Table 1. Evidently, considerable fine scale information has been dredged up by this particular technique. It may be unwise to dismiss that information as noise. In the lower right images in Figures 1 and 2, there are vertical and horizontal lines that criss-cross the fingerprint, as well as various other features that are not easily discernible in the forensic Photoshop image. Some of these details may be significant. At the very least, the histogram equalized image alerts the forensic analyst to the possibly important fine structure present in the original image, information that is not recovered by the Photoshop process.

Figure 3 shows additional enhancements applied to the original print (A), using IDL Sobel filtering, IDL unsharp making, and IDL adaptive histogram equalization. The corresponding reverse images are shown in Figure 4 on the adjoining page. Here, and throughout this report, intensities were artificially increased after Sobel filtering and unsharp masking, to compensate for the faint imagery that generally results from applying these two techniques. The image gradient norms in Table 2 for the IDL Sobel and IDL unsharp masking images, are in general agreement with those for the Photoshop and MATLAB contrast adjusted images in Table 1. In fact, these four enhanced images and their reversed counterparts, are roughly of the same general quality, although important differences exist and can be detected by the trained eye. On the other hand, the IDL adaptive histogram equalized image in Figure 3 is distinctly different from the other five enhancements in image (A). Despite the unwelcome amount of background detail in the IDL adaptive case, a higher quality reconstruction of the actual fingerprint has been realized in Figure 3, and in the counterpart reverse image in Figure 4. This is accompanied by larger $L^{1}$ and $L^{2}$ image and image gradient norms, than was the case in the MATLAB non adaptive case in Figure 1.

Clearly, a more complete picture emerges of the fingerprint in latent print (A), when the forensic Photoshop processed image in Figure 1 is complemented by the alternative independent enhancements displayed in Figures 1 through 4.

Figures 5 through 8 deal with latent print (B). Here, the accompanying forensic Photoshop processed image is of poor quality, as shown in Figure 5. Significant improvement is provided by the MATLAB enhancements in Figures 5 and 6 , with the histogram equalized image providing the most information. Again, note the presence of horizontal and verical streaks that criss-cross the lower right images in Figures 5 and 6 , as well as some other features not evident in the other images in Figures 5 and 6. The IDL Sobel and unsharp masking images in Figures 7 and 8 appear less sharp than the MATLAB contrast adjusted image in Figures 5 and 6 . These two IDL images have approximately the same value for $\|\nabla f\|_{1}$ as has the MATLAB contrast adjusted image, but the latter image has a significantly larger value for $\|\nabla f\|_{2}$. A similar divergence between $L^{1}$ and $L^{2}$ norms was already observed in the discussion of latent print (A). The IDL adaptive histogram equalized images in Figures 7 and 8 may not appear helpful when viewed on the printed page. However, when viewed on a high resolution device, such as an active matrix, backlit, LCD monitor, these IDL adaptive images are seen to contain higher quality information than is available 
in the MATLAB non adaptive case in Figures 5 and 6 . In summary, in this difficult latent print, substantially more useful information can be obtained using the above alternative enhancements, than was provided in the forensic Photoshop processed image.

Behavior in latent print (C), illustrated in Figures 9 through 12, is quite similar to that in latent print (A). The MATLAB contrast adjusted image is again sharper than the forensic Photoshop image. This is picked up in the $\|\nabla f\|_{2}$ norm in Table 5 , but not in the $\|\nabla f\|_{1}$ norm. The MATLAB histogram equalized image recovers potentially significant information that is not visible in the Photoshop enhancement. This is confirmed by the substantially larger image gradient norms. The IDL Sobel and IDL unsharp masking images are of roughly the same quality as the forensic Photoshop image, with approximately the same image gradient norms. Finally, in Figures 11 and 12, despite the distracting amount of background detail, the IDL adaptive equalized images, viewed on a modern LCD monitor, provide more useful information in the lower half of latent print $(\mathrm{C})$ than do the non adaptive MATLAB images in Figures 9 and 10.

5. Concluding remarks and remaining questions. For the class of latent fingerprints represented by the above three exmples, Sobel filtering and unsharp masking tend to produce enhancements of the same general quality as the forensic Photoshop process. However, contrast adjustment using MATLAB imadjust, or IDL bytscl, typically produces higher quality images, accompanied by larger values for $\|\nabla f\|_{2}$.

Histogram equalization using MATLAB histeq, or IDL hist_equal, produces a different category of enhancement, with significantly larger values for $\|\nabla f\|_{2}$, along with recovery of potentially significant fine scale information typically not present in the forensic Photoshop processed image. Adaptive histogram equalization, using IDL adapt_hist_equal, tends to bring up considerable background detail, while producing higher quality fingerprint reconstructions than may be feasible with non-adaptive equalization. In the case of latent print (A) for example, the dark patches obscuring the fingerprint image in the lower right corner of Figure 2, are not present in the image in the lower right corner of Figure 4.

As previously noted, the alternative enhancements displayed in Figures 1 through 12 resulted from the use of MATLAB and/or IDL commands in their simplest forms, where default values were assumed for the associated optional parameters. It would be instructive to explore the same enhancement methods with more sophisticated parameter choices.

A question of major interest is whether the additional information provided in some of these alternative enhancements leads to the same matches obtained using the forensic Photoshop process. If that is not the case, how much smoothing of the new enhancement is necessary to recover the Photoshop induced matches?

An instructive line of inquiry would start with a histogram equalized image displaying substantial fine scale detail. Subjecting that image to systematic 'slow motion' fractional diffusion denoising [7], would create a sequence of gradually smoother enhancements. How would the resulting matches vary with the different images along that sequence?

\section{REFERENCES}

[1] F. McRoberts and S. Mills, Digitized prints can point finger at the innocent, Chicago Tribune, January 32005. 
[2] F. McRoberts and S. Mills, US seeks review of fingerprint techniques, Chicago Tribune, February 212005.

[3] Image Processing Toolbox User's Guide (2012), The MathWorks, Inc. Natick, MA 01760.

[4] ITTVIS Interactive Data Language (IDL), http://www.exelisvis.com/IDL.aspx

[5] PV-Wave 7.5 Image Processing Toolkit User's Guide, Visual Numerics, Inc. Houston, TX 77042 .

[6] W. K. Pratt, Digital Image Processing, (2012), Wiley-Interscience, New York.

[7] A. S. Carasso AND A. E. Vladár, Fractional diffusion, low exponent Lévy stable laws, and 'slow motion' denoising of Helium Ion microscope nanoscale imagery, Journal of Research of the National Institute of Standards and Technology, Volume 117 (2012), http://dx.doi.org/10.6028/jres.117.006 


\section{ALTERNATIVE ENHANCEMENTS OF LATENT PRINT (A)}

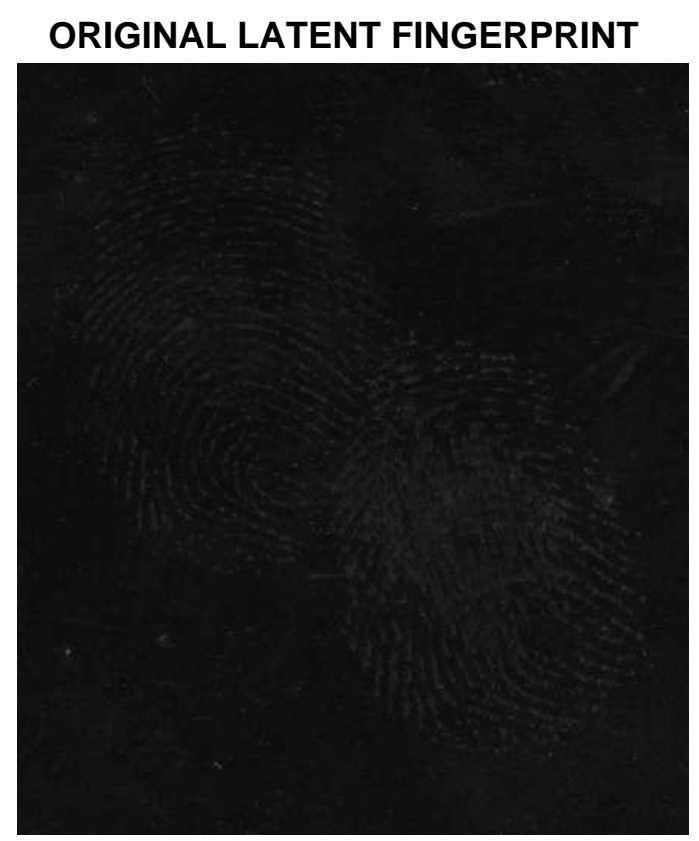

\section{FORENSIC PHOTOSHOP PROCESS}

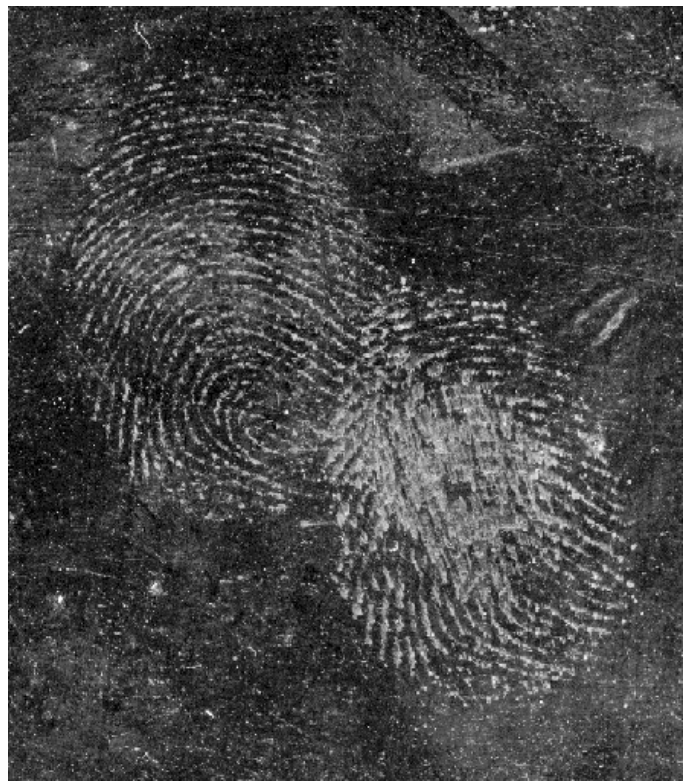

MATLAB CONTRAST ADJUSTMENT
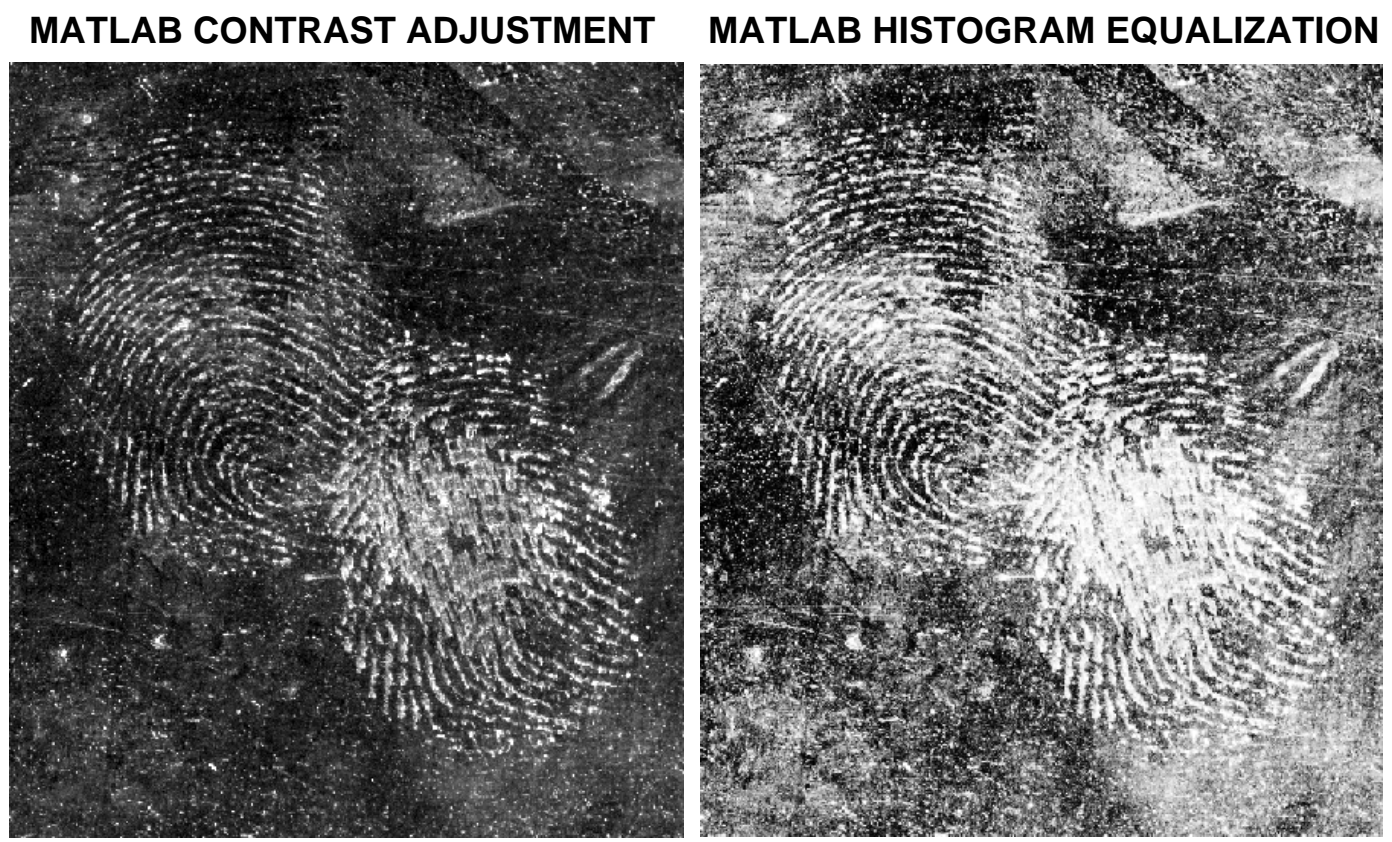

FIG. 1. Enhancements of latent fingerprint (A). Original print, accompanying forensic Photoshop enhancement, and two alternative MATLAB enhancements. Significant enhancement of background features is apparent in histogram equalized image. This is reflected in the image gradient norms in Table 1.

TABLE 1.

Image metrics in enhancements of latent print $(A)$.

\begin{tabular}{|c|c|c|c|c|}
\hline Image $f(x, y)$ & $\|f\|_{1}$ & $\|f\|_{2}$ & $\|\nabla f\|_{1}$ & $\|\nabla f\|_{2}$ \\
\hline Original Latent Print (A) & 16 & 18 & 2600 & 3900 \\
\hline Forensic Photoshop (A) & 61 & 75 & 25000 & 36000 \\
\hline Matlab Contrast Adj. (A) & 60 & 80 & 25000 & 40000 \\
\hline Matlab Histogram Eq. (A) & 112 & 138 & 42000 & 60000 \\
\hline
\end{tabular}




\section{REVERSE IMAGES IN ENHANCED LATENT PRINT (A)}

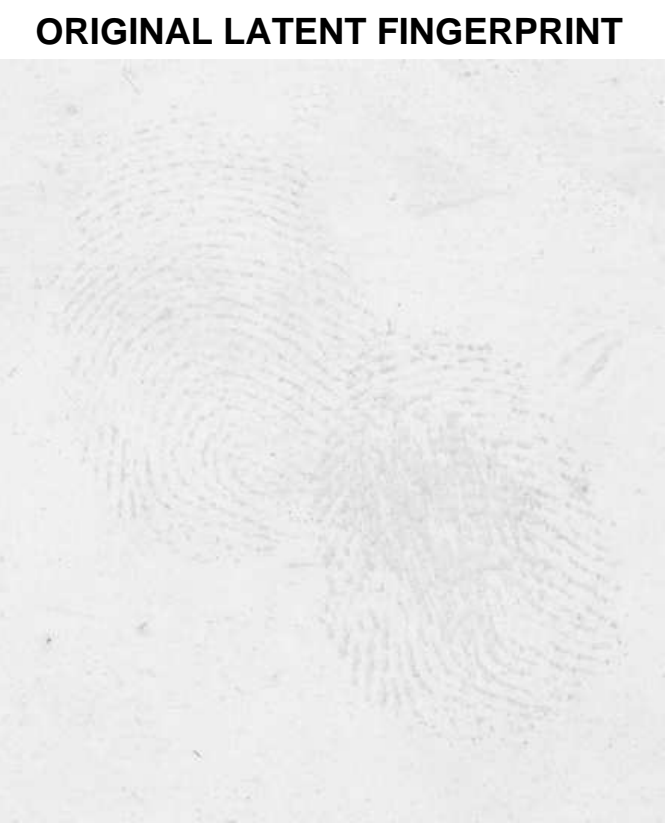

\section{FORENSIC PHOTOSHOP PROCESS}

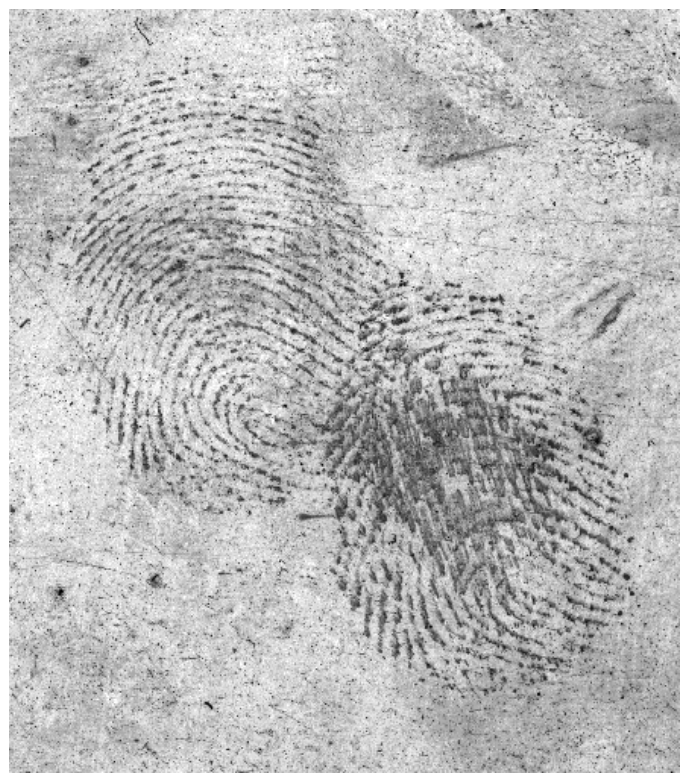

\section{MATLAB CONTRAST ADJUSTMENT}
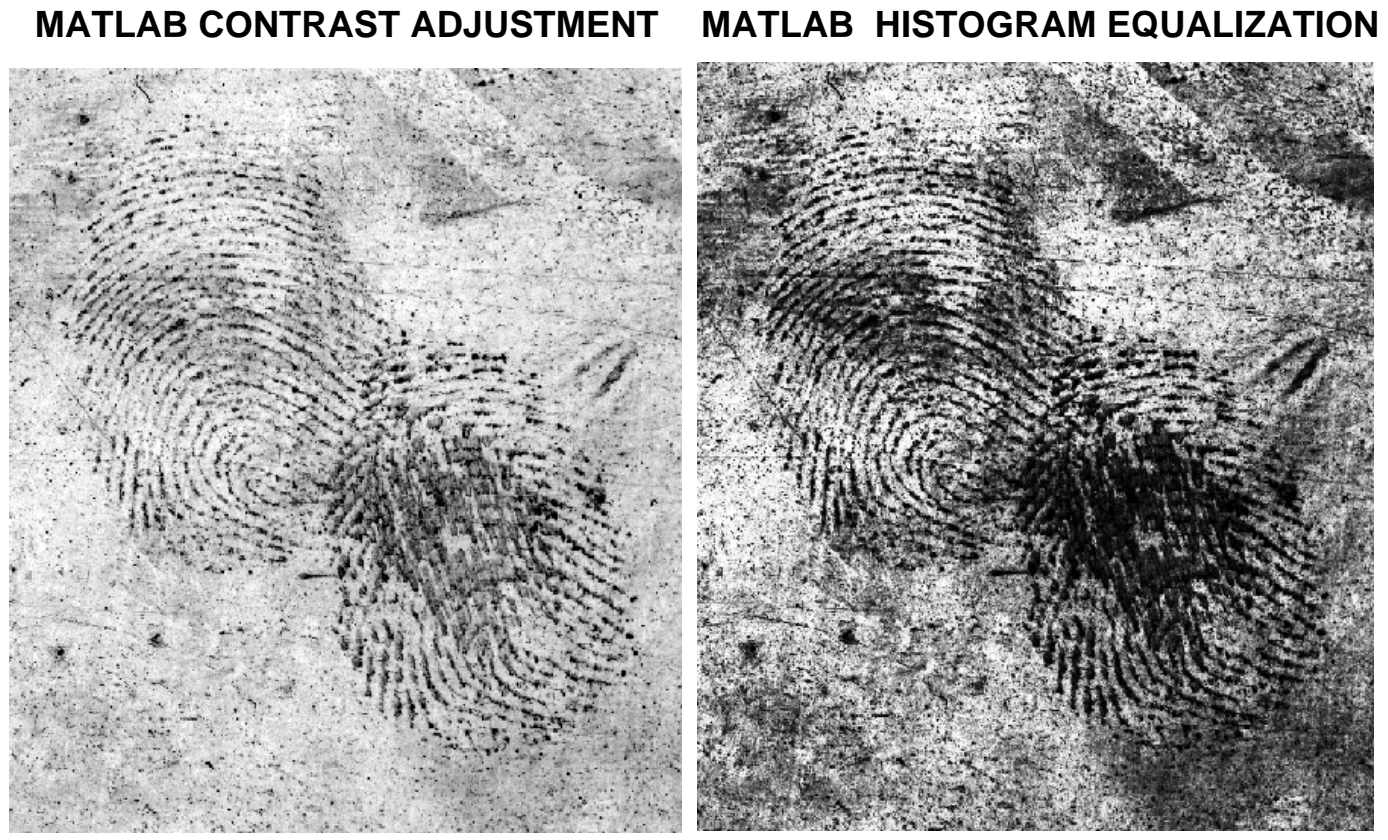

FIG. 2. Reverse images in the latent print (A) enhancements shown in Figure 1. 


\section{ADDITIONAL ENHANCEMENTS OF LATENT PRINT (A)}

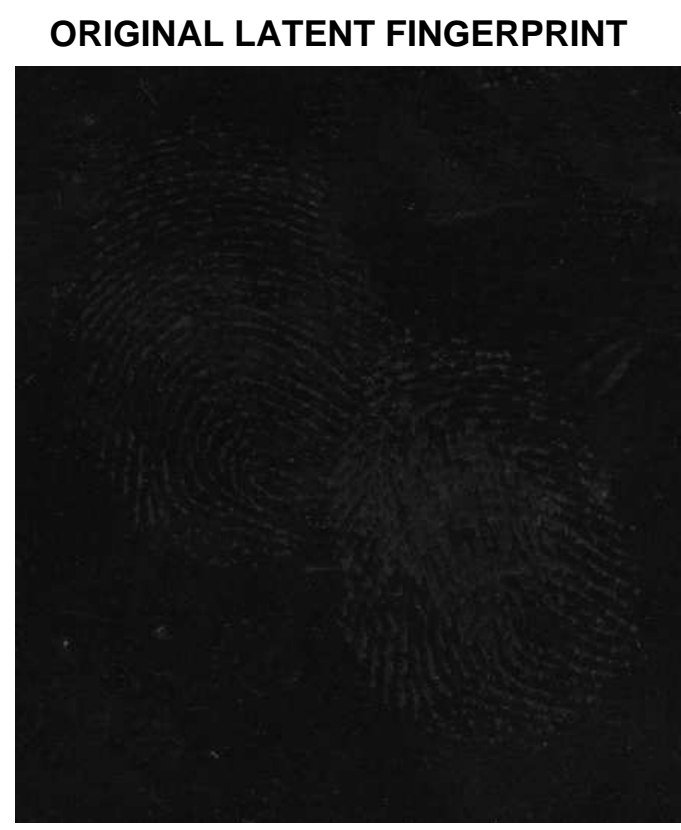

IDL SOBEL FILTERING ( x 2)
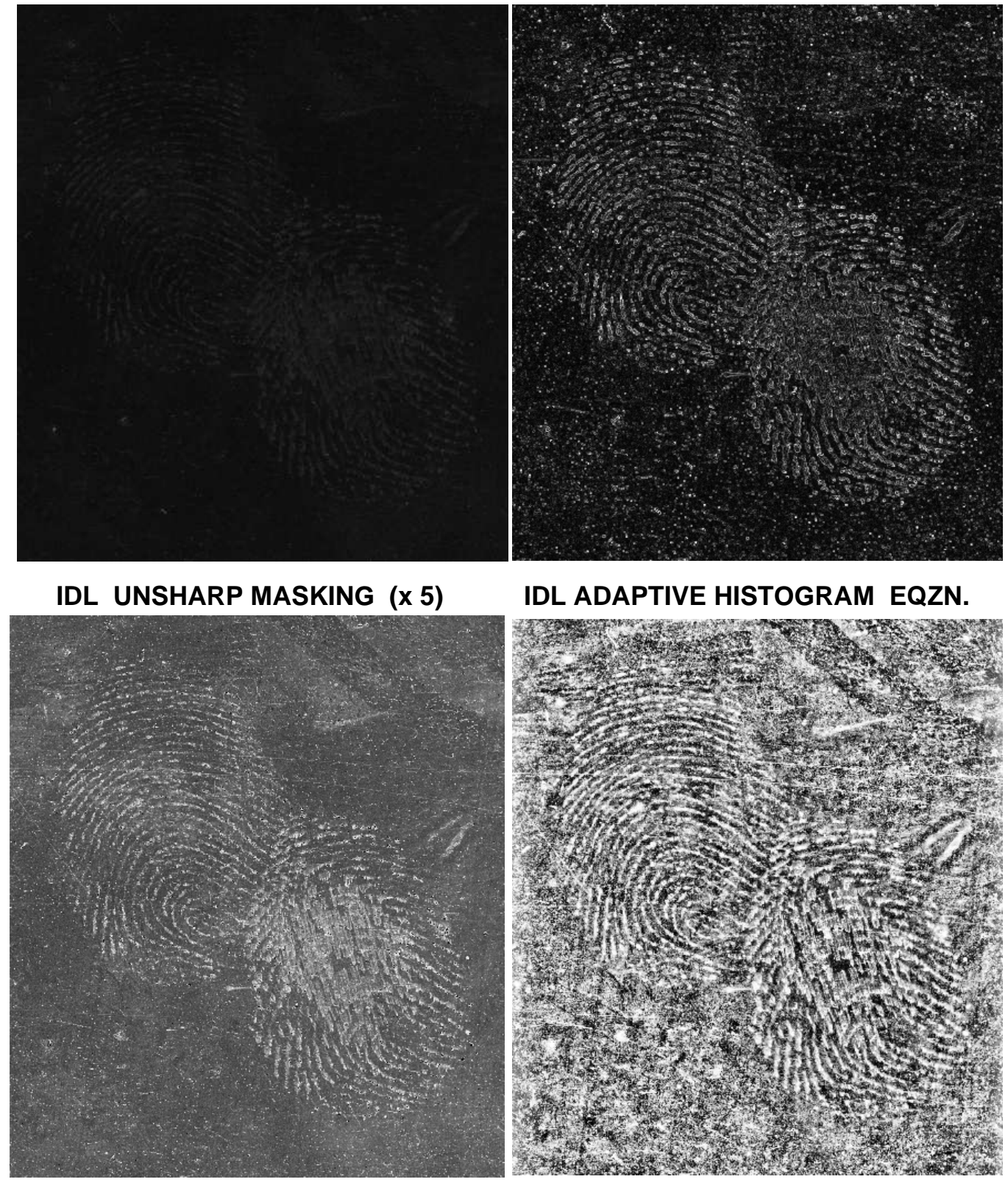

IDL ADAPTIVE HISTOGRAM EQZN.

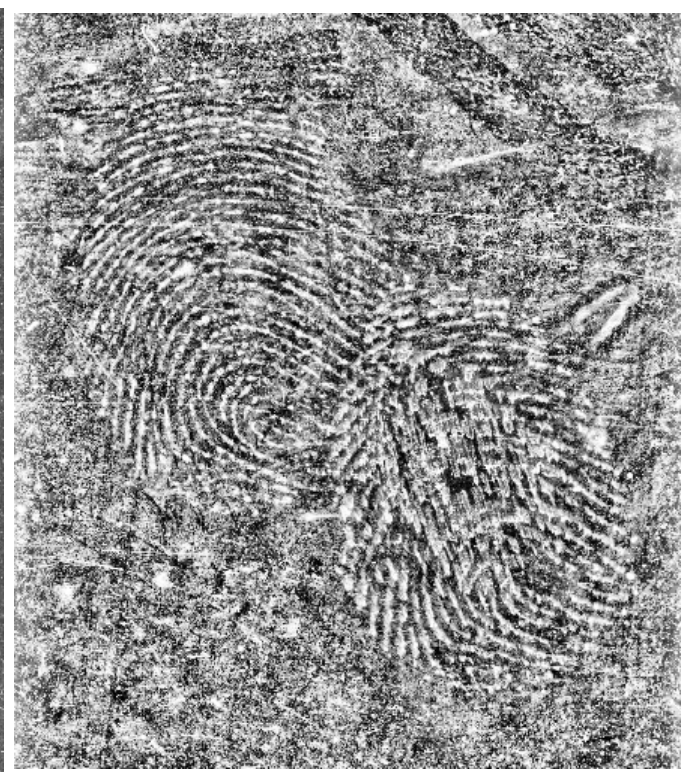

FIG. 3. Additional enhancements of latent fingerprint (A). Original print and three alternative IDL enhancements. Intensities were doubled after Sobel filtering to improve visibility. Similarly, intensities were quintupled after unsharp masking. Noticeably different adaptive histogram equalized image displays considerable background information. This is reflected in the larger image gradient norms in Table 2.

TABLE 2.

Image metrics in additional enhancements of latent print (A).

\begin{tabular}{|c|c|c|c|c|}
\hline Image $f(x, y)$ & $\|f\|_{1}$ & $\|f\|_{2}$ & $\|\nabla f\|_{1}$ & $\|\nabla f\|_{2}$ \\
\hline Original Latent Print (A) & 16 & 18 & 2600 & 3900 \\
\hline IDL Sobel Filtering (×2) (A) & 31 & 45 & 24000 & 35000 \\
\hline IDL Unsharp Mask (×5) (A) & 86 & 97 & 24000 & 37000 \\
\hline IDL Adaptive Hist. Eq. (A) & 127 & 150 & 54000 & 70000 \\
\hline
\end{tabular}




\section{REVERSE IMAGES IN ENHANCED LATENT PRINT (A)}
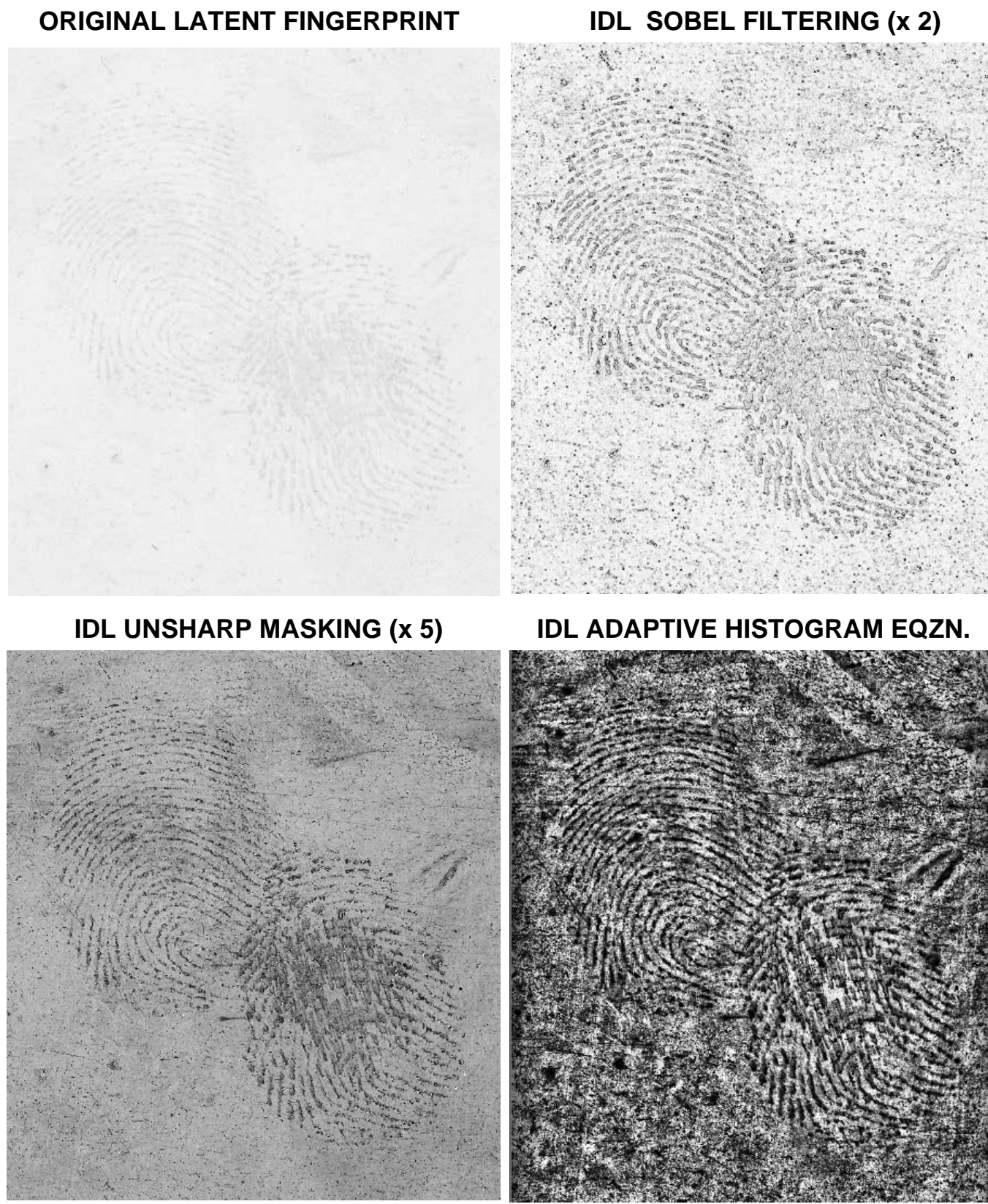

IDL ADAPTIVE HISTOGRAM EQZN.

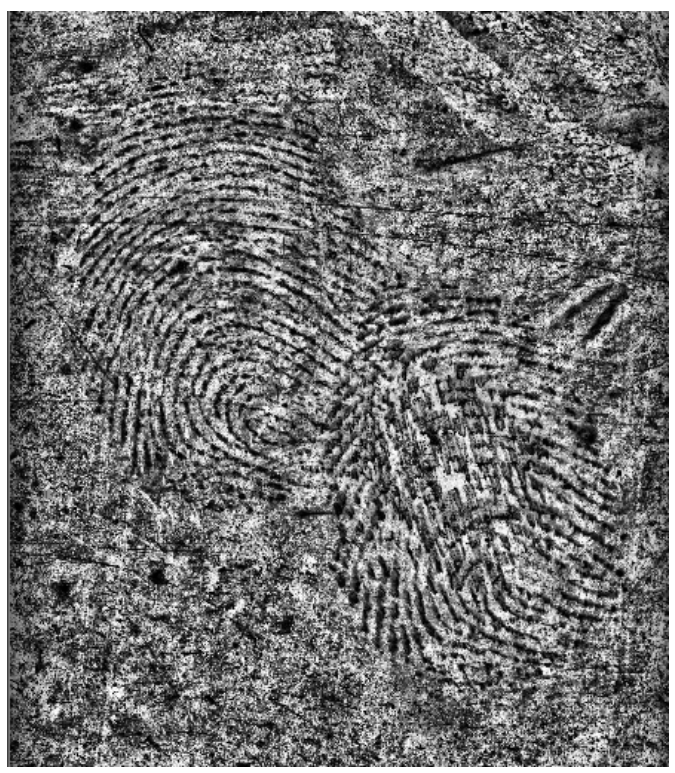

FIG. 4. Reverse images in the additional latent print (A) enhancements shown in Figure 3. 


\title{
ALTERNATIVE ENHANCEMENTS OF LATENT PRINT (B)
}

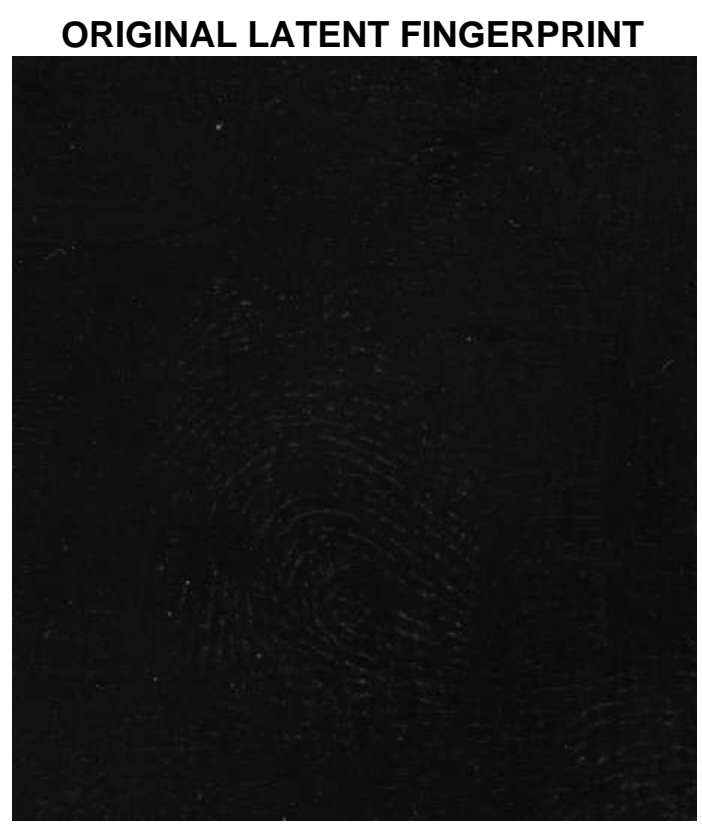

\author{
FORENSIC PHOTOSHOP PROCESS
}

MATLAB CONTRAST ADJUSTMENT

MATLAB HISTOGRAM EQUALIZATION
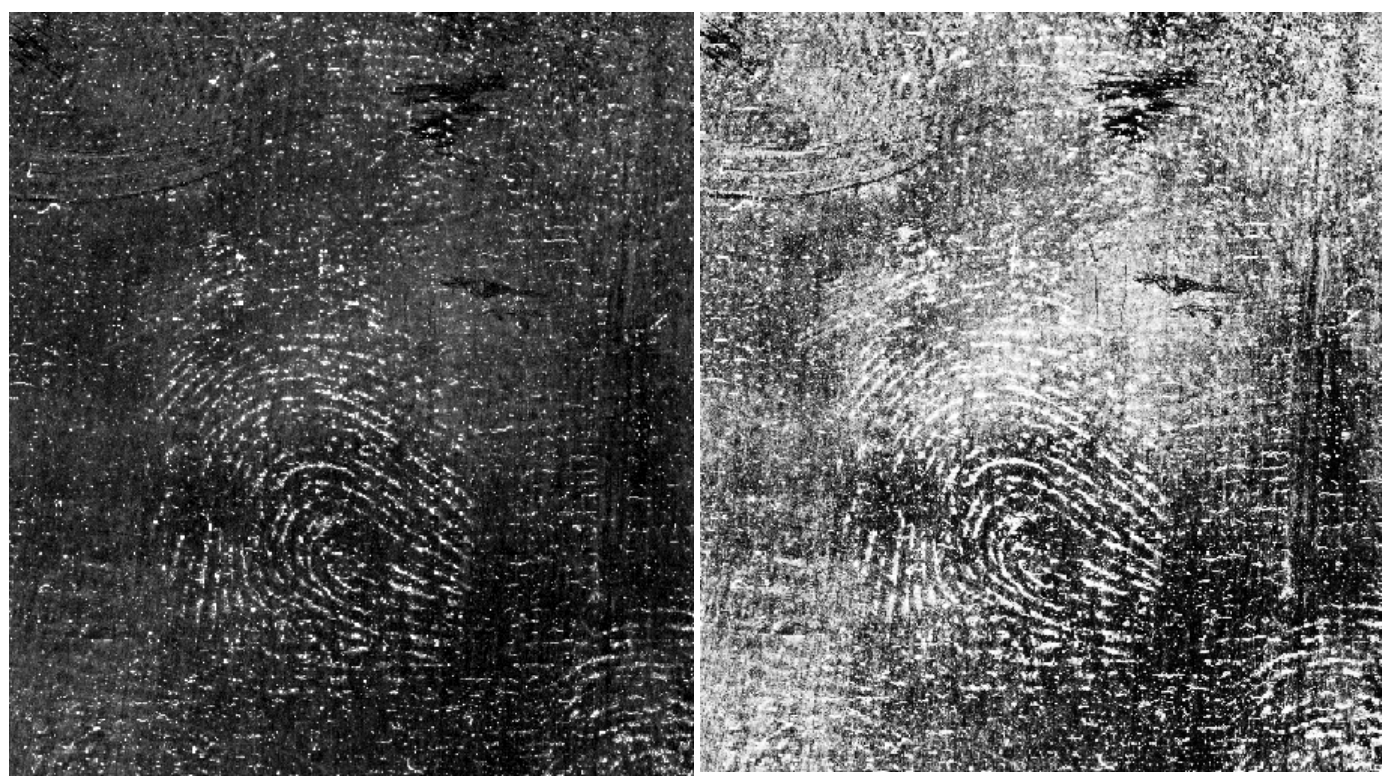

FIG. 5. Enhancements of latent fingerprint (B). Original print, accompanying forensic Photoshop enhancement, and two alternative MATLAB enhancements. As in Figure 1, histogram equalized image shows substantial small scale background features, and has larger image gradient norms.

\section{TABLE 3.}

Image metrics in enhancements of latent print (B).

\begin{tabular}{|c|c|c|c|c|}
\hline Image $f(x, y)$ & $\|f\|_{1}$ & $\|f\|_{2}$ & $\|\nabla f\|_{1}$ & $\|\nabla f\|_{2}$ \\
\hline Original Latent Print (B) & 15 & 16 & 2500 & 4200 \\
\hline Forensic Photoshop (B) & 29 & 33 & 8000 & 12000 \\
\hline Matlab Contrast Adj. (B) & 52 & 69 & 26000 & 43000 \\
\hline Matlab Histogram Eq. (B) & 115 & 140 & 49000 & 69000 \\
\hline
\end{tabular}




\section{REVERSE IMAGES IN ENHANCED LATENT PRINT (B)}

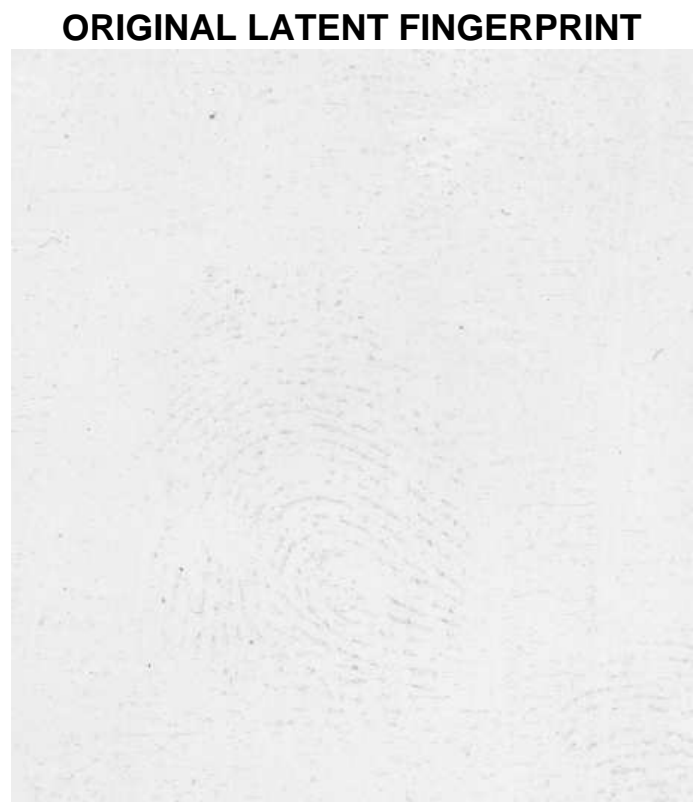

\section{FORENSIC PHOTOSHOP PROCESS}
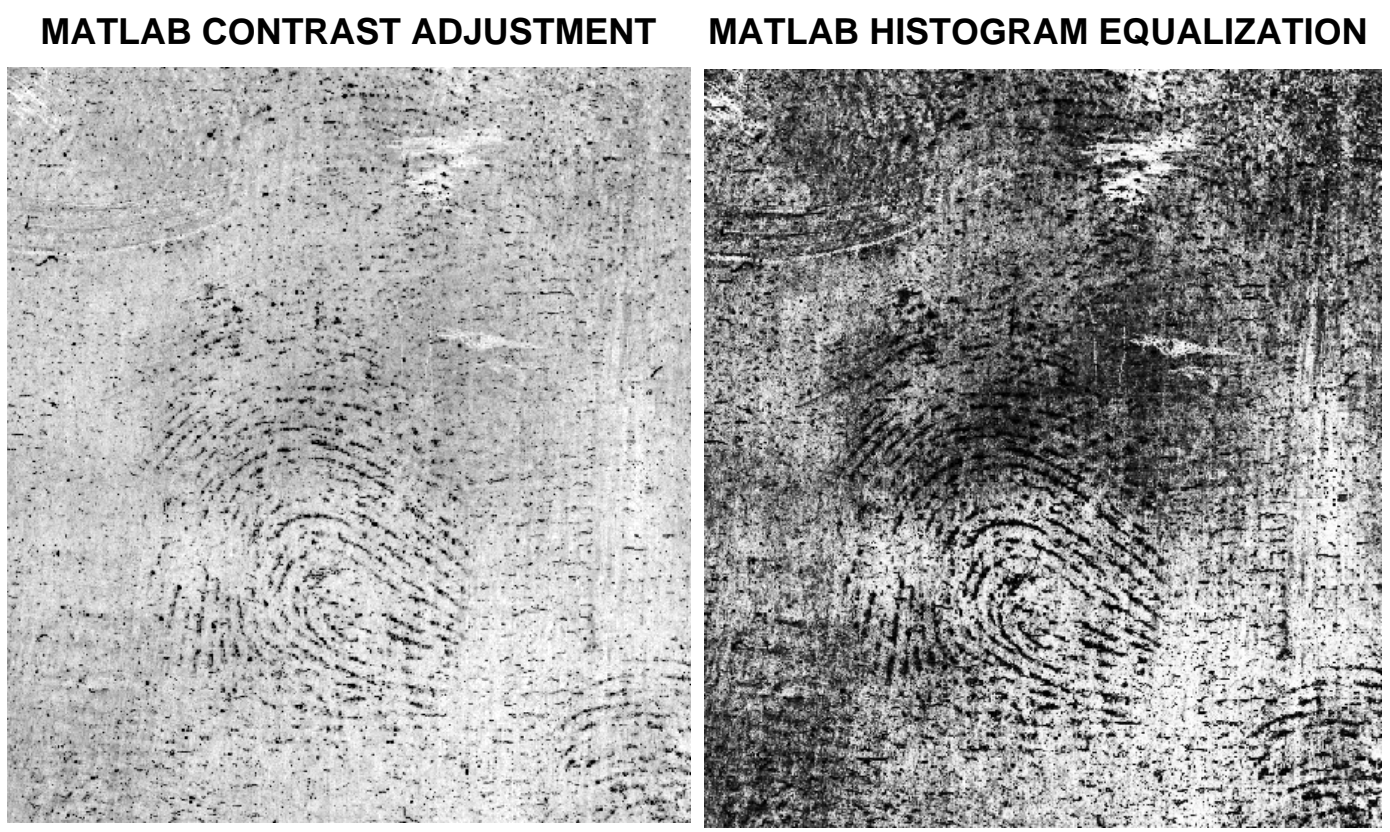

FIG. 6. Reverse images in the latent print (B) enhancements shown in Figure 5 


\section{ADDITIONAL ENHANCEMENTS OF LATENT PRINT (B)}
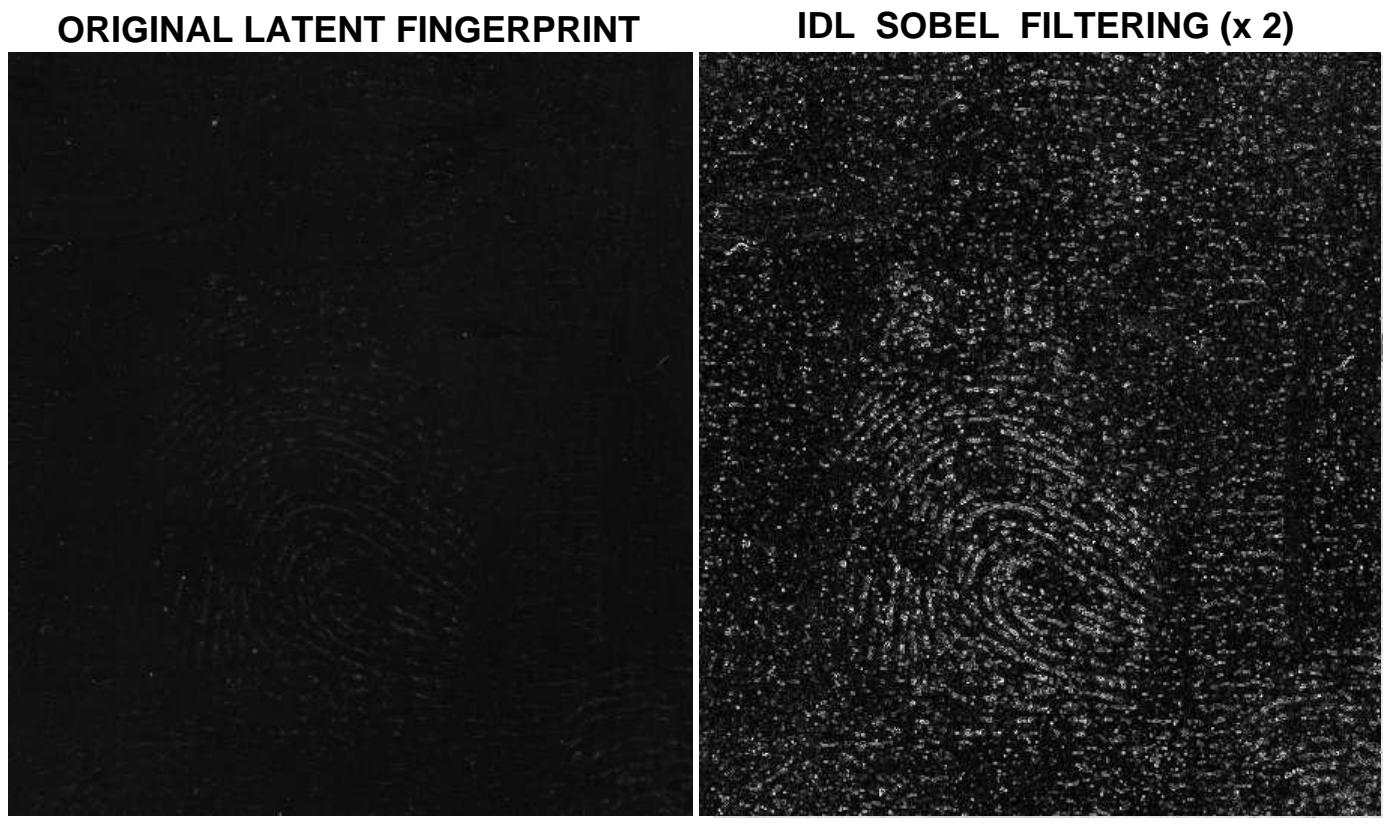

IDL UNSHARP MASKING (x 5)

IDL ADAPTIVE HISTOGRAM EQZN.
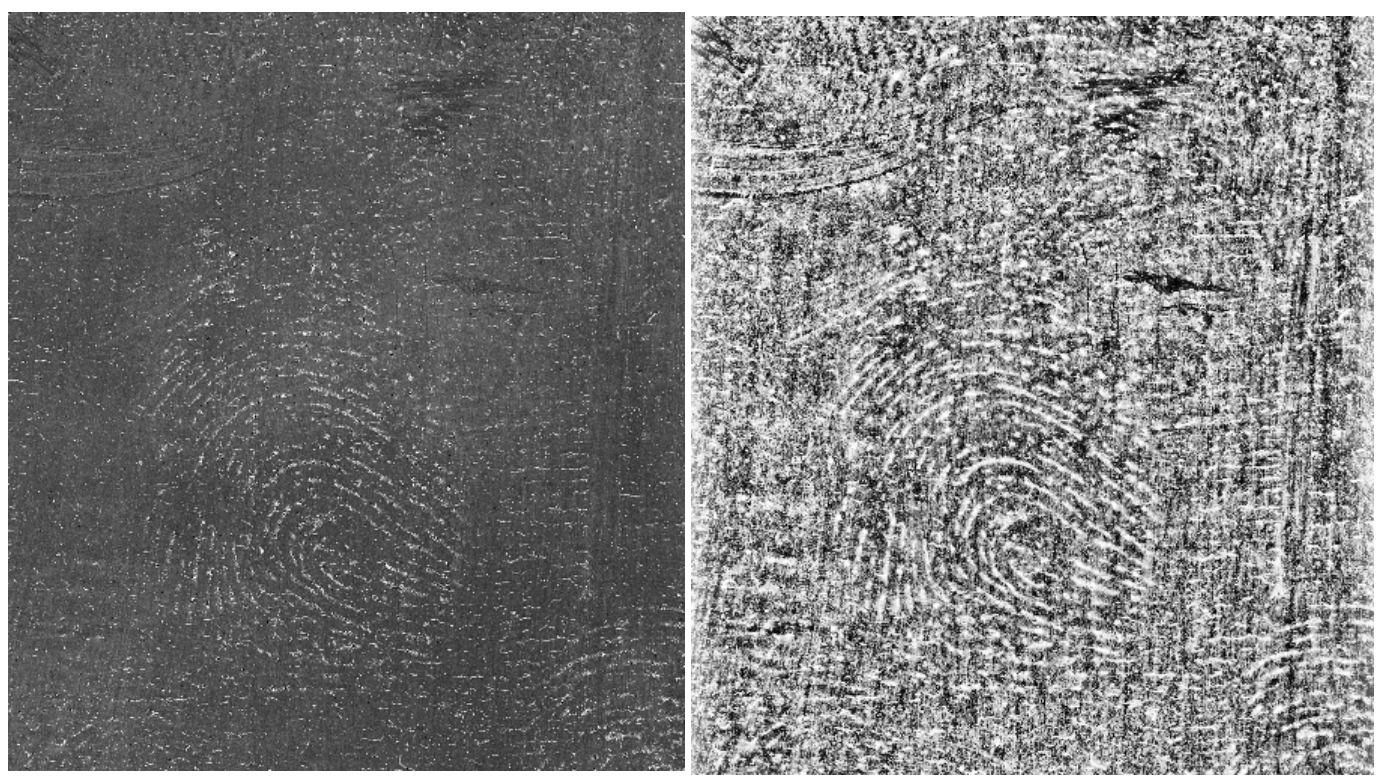

FIG. 7. Additional enhancements of latent fingerprint (B). Original print and three alternative IDL enhancements. As in Figure 3, intensities were doubled after Sobel filtering, and quintupled after unsharp masking. Distinctly different adaptive histogram equalized image displays considerable small scale detail, as is confirmed by the larger image gradient norms in Table 4.

\section{TABLE 4.}

Image metrics in additional enhancements of latent print (B).

\begin{tabular}{|c|c|c|c|c|}
\hline Image $f(x, y)$ & $\|f\|_{1}$ & $\|f\|_{2}$ & $\|\nabla f\|_{1}$ & $\|\nabla f\|_{2}$ \\
\hline Original Latent Print (B) & 15 & 16 & 2500 & 4200 \\
\hline IDL Sobel Filtering $(\times 2)(\mathrm{B})$ & 27 & 43 & 24000 & 38000 \\
\hline IDL Unsharp Mask (×5) (B) & 79 & 87 & 23000 & 37000 \\
\hline IDL Adaptive Hist. Eq. (B) & 135 & 156 & 62000 & 79000 \\
\hline
\end{tabular}




\section{REVERSE IMAGES IN ENHANCED LATENT PRINT (B)}

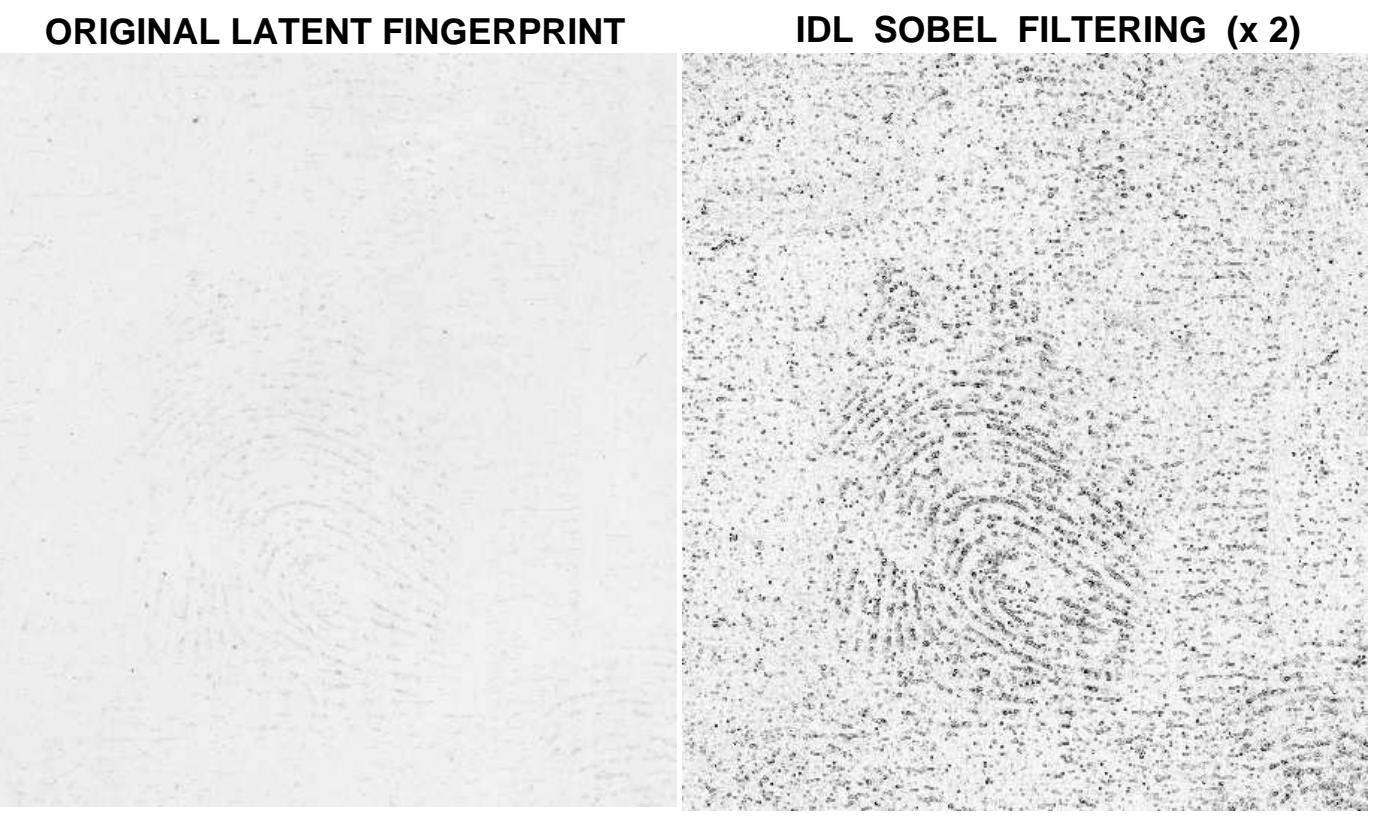

IDL UNSHARP MASKING (x 5)

IDL ADAPTIVE HISTOGRAM EQZN.

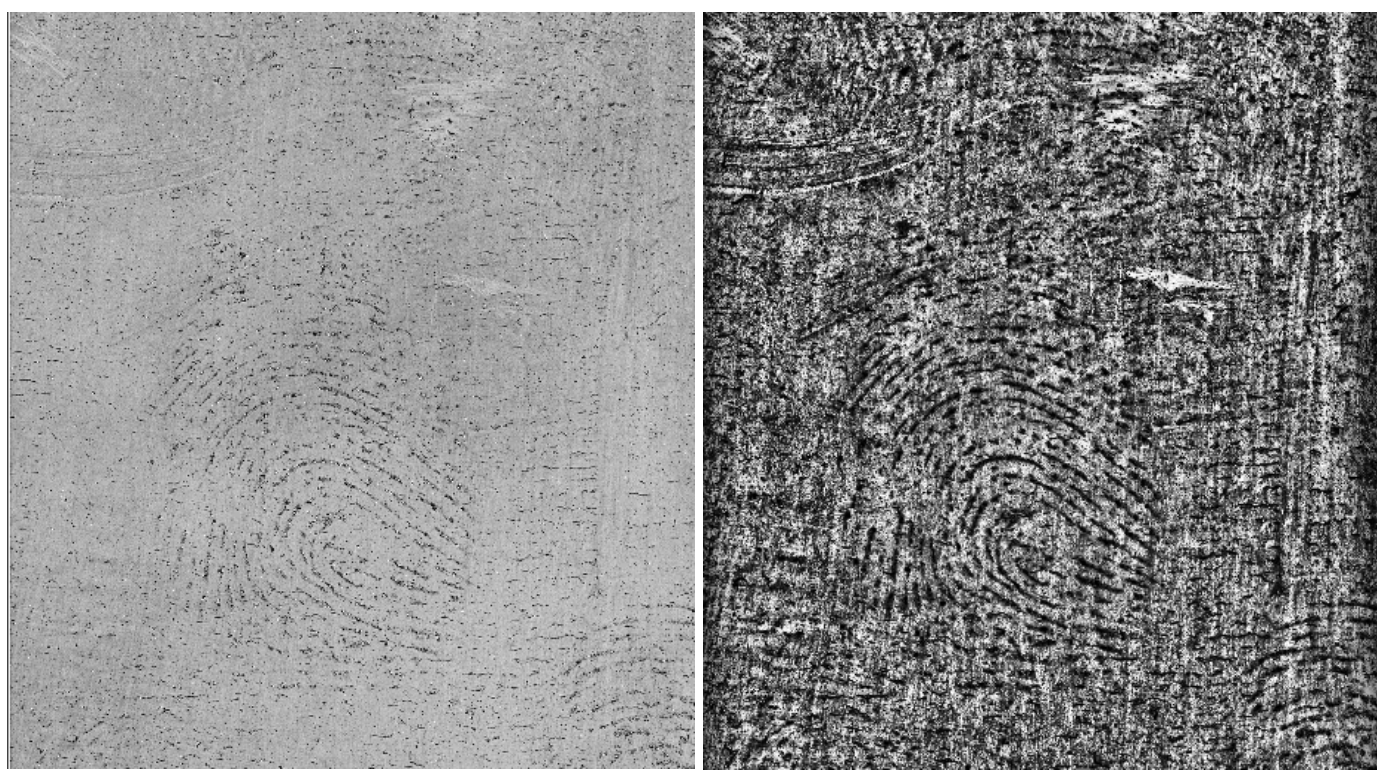

FIG. 8. Reverse images in the additional latent print (B) enhancements shown in Figure $\%$ 


\section{ALTERNATIVE ENHANCEMENTS OF LATENT PRINT (C)}

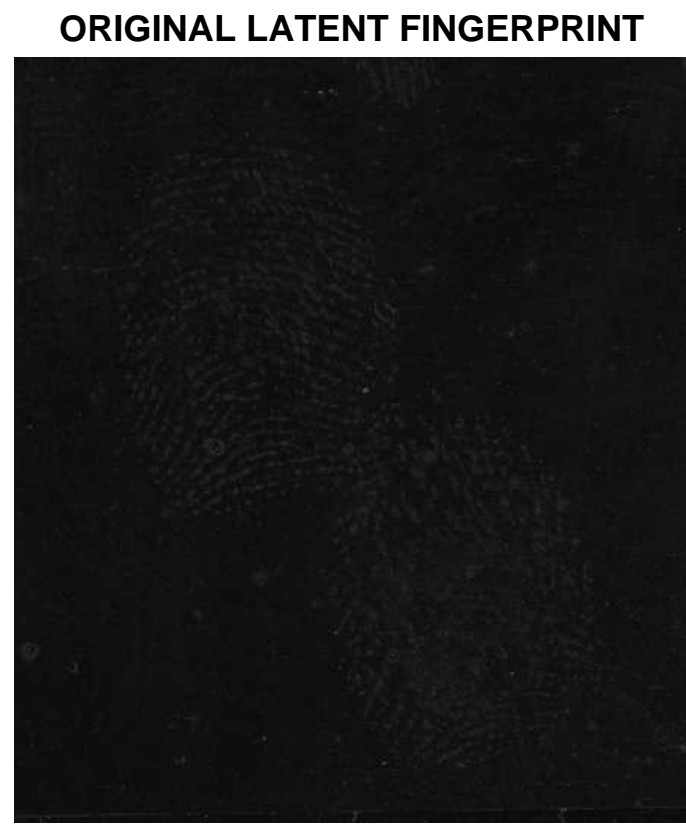

FORENSIC PHOTOSHOP PROCESS
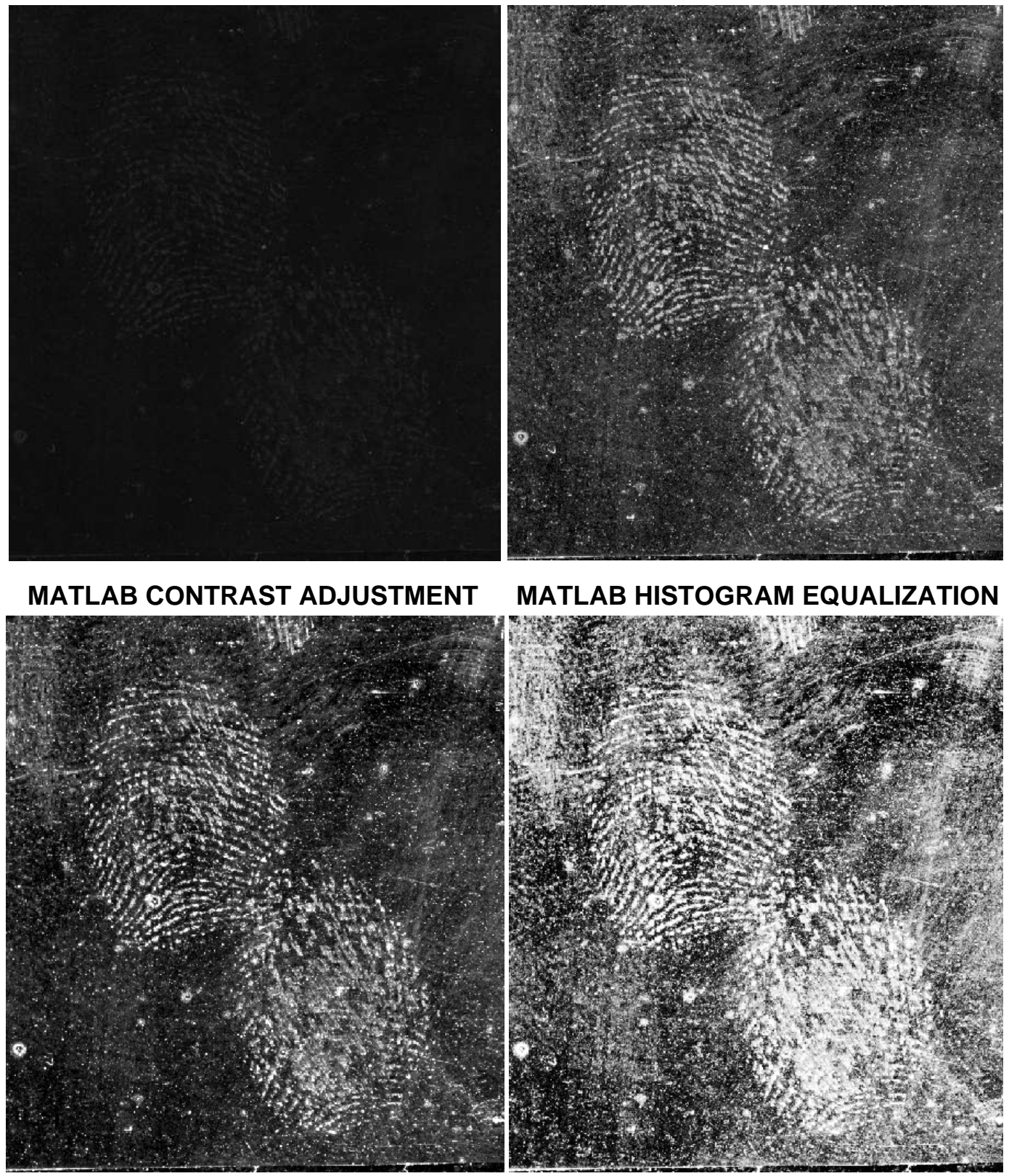

MATLAB HISTOGRAM EQUALIZATION

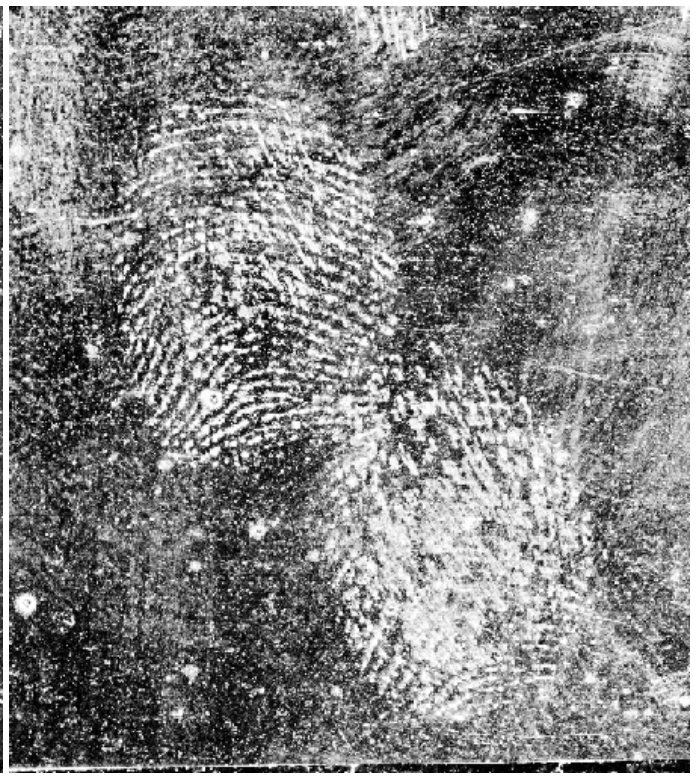

FIG. 9. Enhancements of latent fingerprint $(C)$. Original print, accompanying forensic Photoshop enhancement, and two alternative MATLAB enhancements. As in Figure 1, histogram equalized image shows substantial small scale background features, and has larger image gradient norms.

TABLE 5.

Image metrics in enhancements of latent print $(C)$.

\begin{tabular}{|c|c|c|c|c|}
\hline Image $f(x, y)$ & $\|f\|_{1}$ & $\|f\|_{2}$ & $\|\nabla f\|_{1}$ & $\|\nabla f\|_{2}$ \\
\hline Original Latent Print (C) & 17 & 18 & 2800 & 4200 \\
\hline Forensic Photoshop (C) & 62 & 76 & 29000 & 37000 \\
\hline Matlab Contrast Adj. (C) & 58 & 78 & 29000 & 44000 \\
\hline Matlab Histogram Eq. (C) & 113 & 139 & 47000 & 65000 \\
\hline
\end{tabular}




\section{REVERSE IMAGES IN ENHANCED LATENT PRINT (C)}

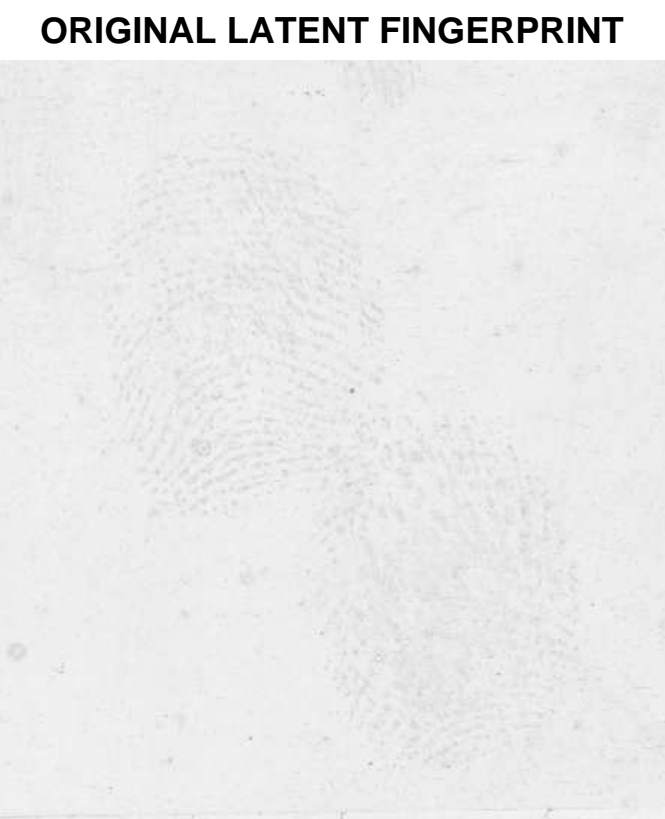

\section{FORENSIC PHOTOSHOP PROCESS}

MATLAB CONTRAST ADJUSTMENT
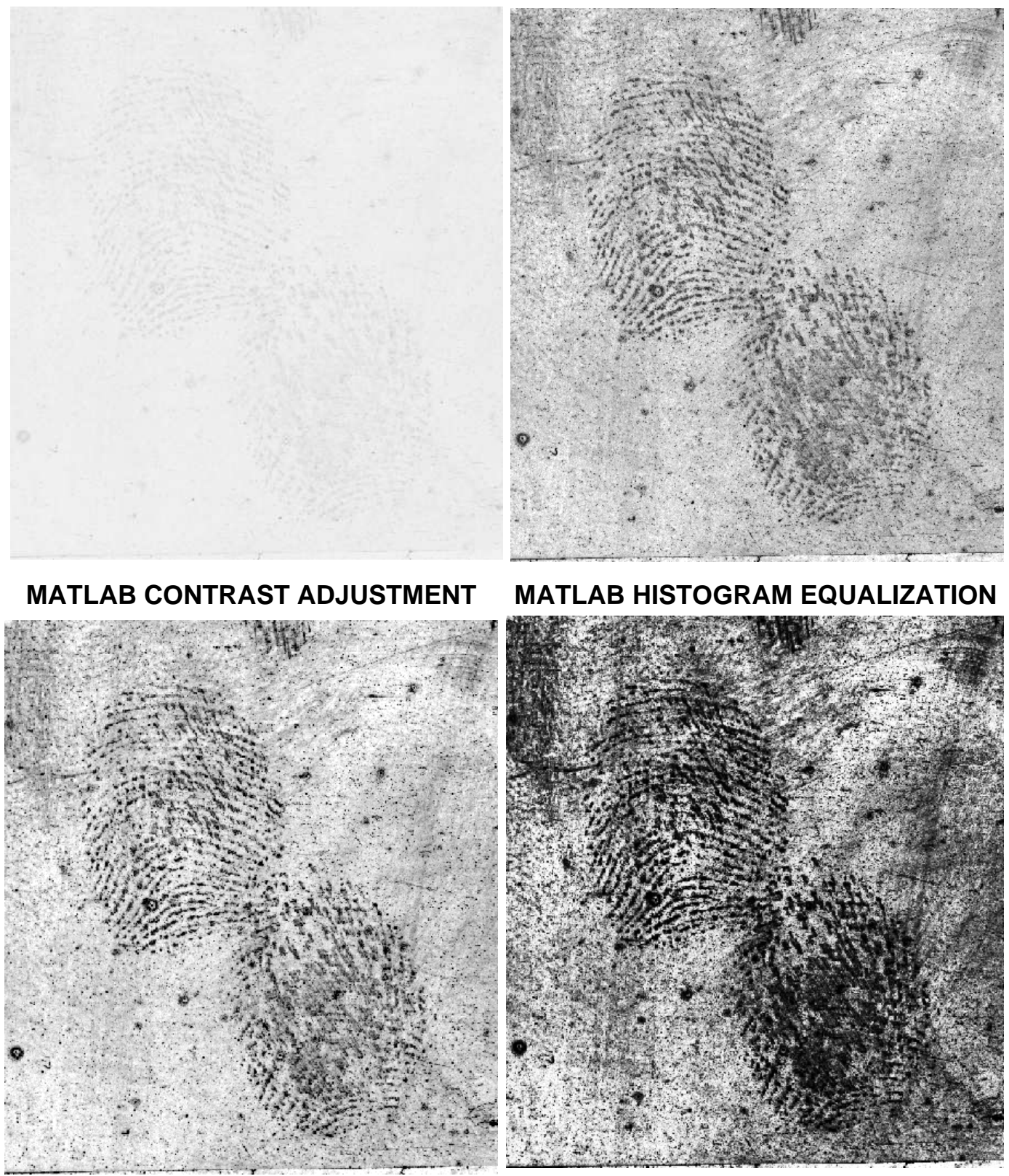

MATLAB HISTOGRAM EQUALIZATION

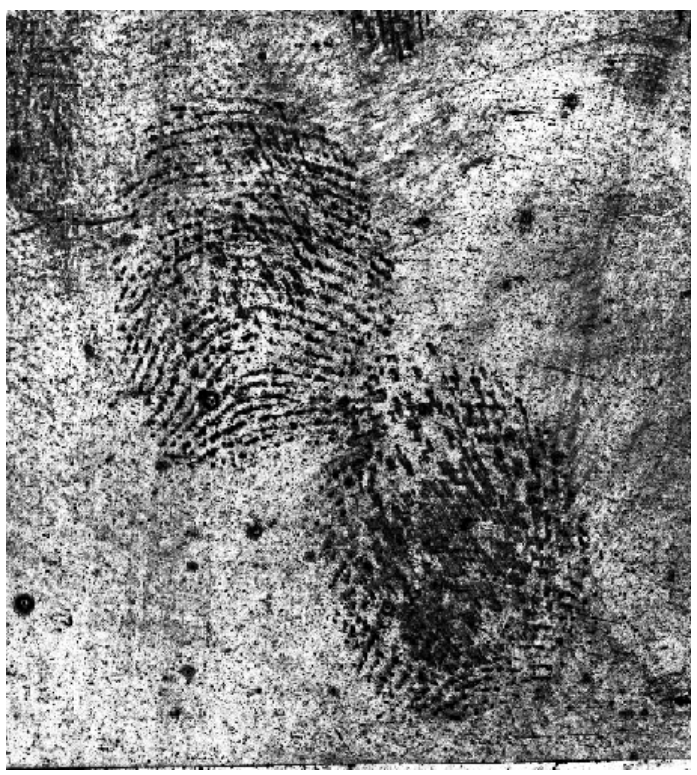

FIG. 10. Reverse images in the latent print $(C)$ enhancements shown in Figure 9. 


\section{ADDITIONAL ENHANCEMENTS OF LATENT PRINT (C)}

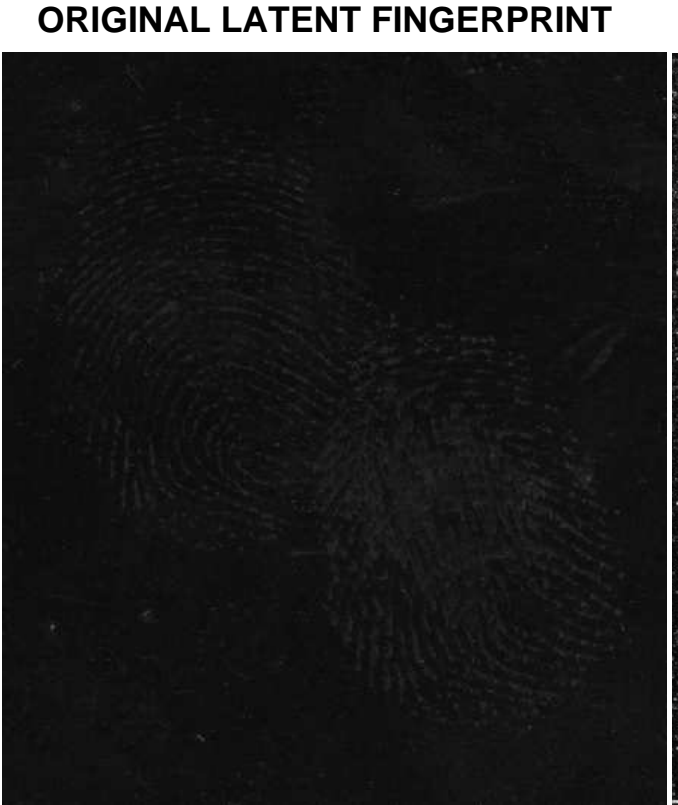

IDL UNSHARP MASKING (x 5)

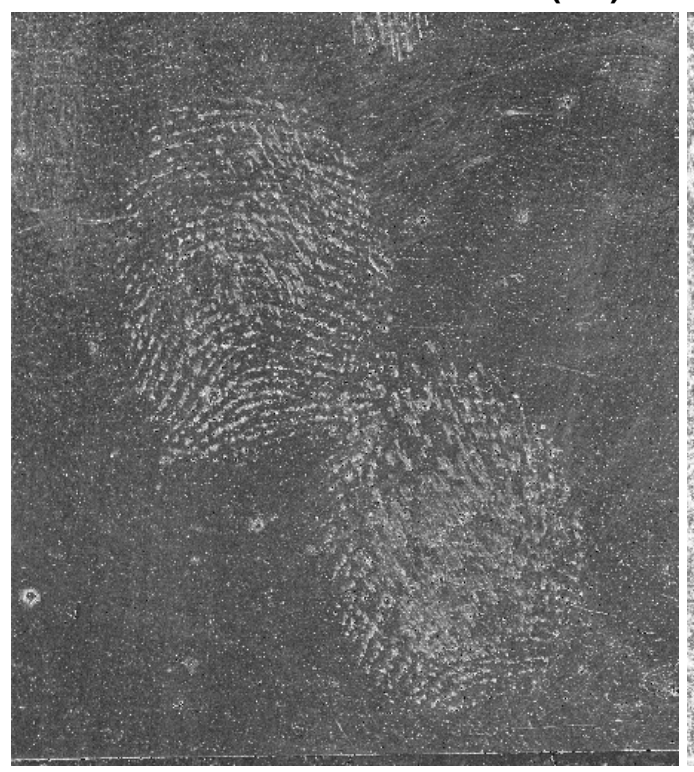

IDL SOBEL FILTERING (x 2)

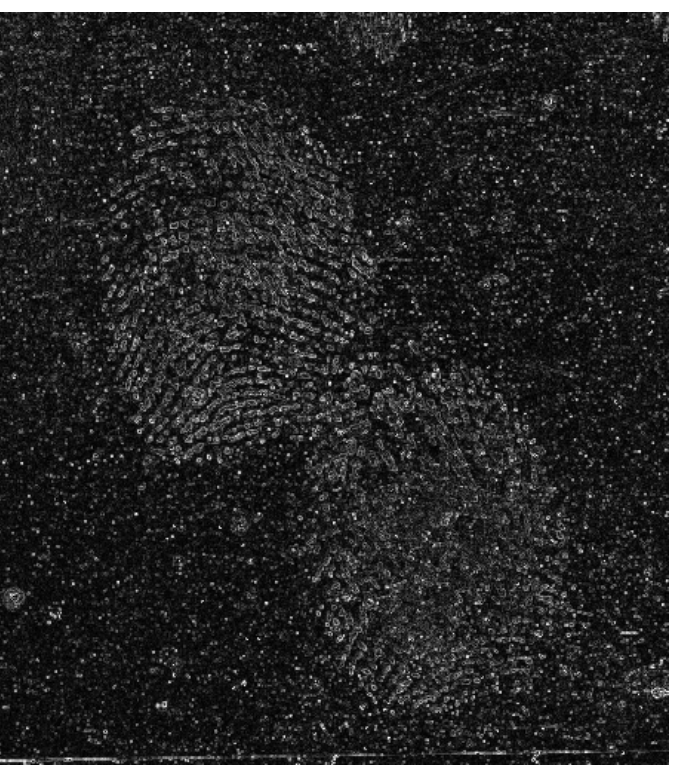

IDL ADAPTIVE HISTOGRAM EQZN.

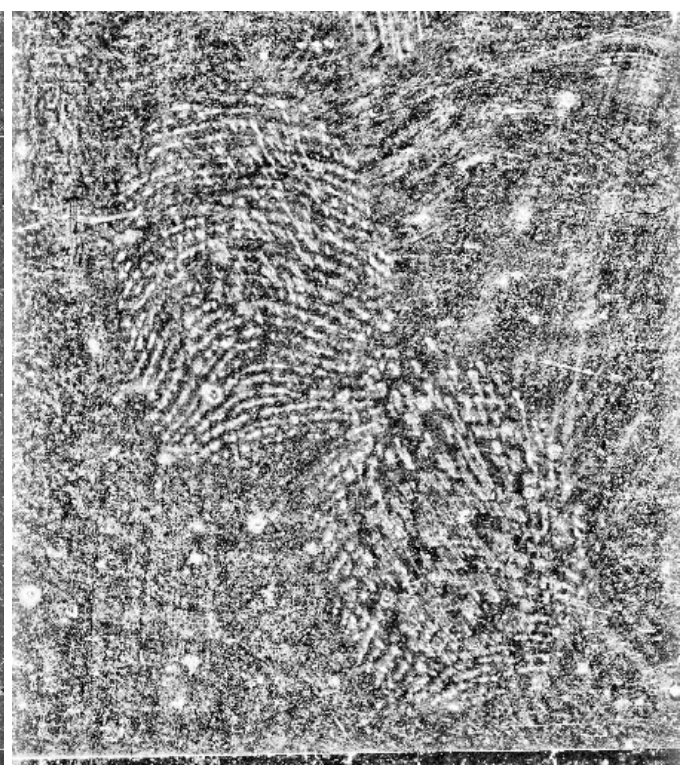

FIG. 11. Additional enhancements of latent fingerprint (C). Original print and three alternative IDL enhancements. As in Figure 3, intensities were doubled after Sobel filtering, and quintupled after unsharp masking. Distinctly different adaptive histogram equalized image displays considerable small scale detail, as is confirmed by the larger image gradient norms in Table 6.

\section{TABLE 6.}

Image metrics in additional enhancements of latent print $(C)$.

\begin{tabular}{|c|c|c|c|c|}
\hline Image $f(x, y)$ & $\|f\|_{1}$ & $\|f\|_{2}$ & $\|\nabla f\|_{1}$ & $\|\nabla f\|_{2}$ \\
\hline Original Latent Print (C) & 17 & 18 & 2800 & 4200 \\
\hline IDL Sobel Filtering (×2) (C) & 31 & 45 & 25000 & 36000 \\
\hline IDL Unsharp Mask (×5) (C) & 88 & 98 & 26000 & 39000 \\
\hline IDL Adaptive Hist. Eq. (C) & 129 & 151 & 59000 & 77000 \\
\hline
\end{tabular}




\section{REVERSE IMAGES IN ENHANCED LATENT PRINT (C)}

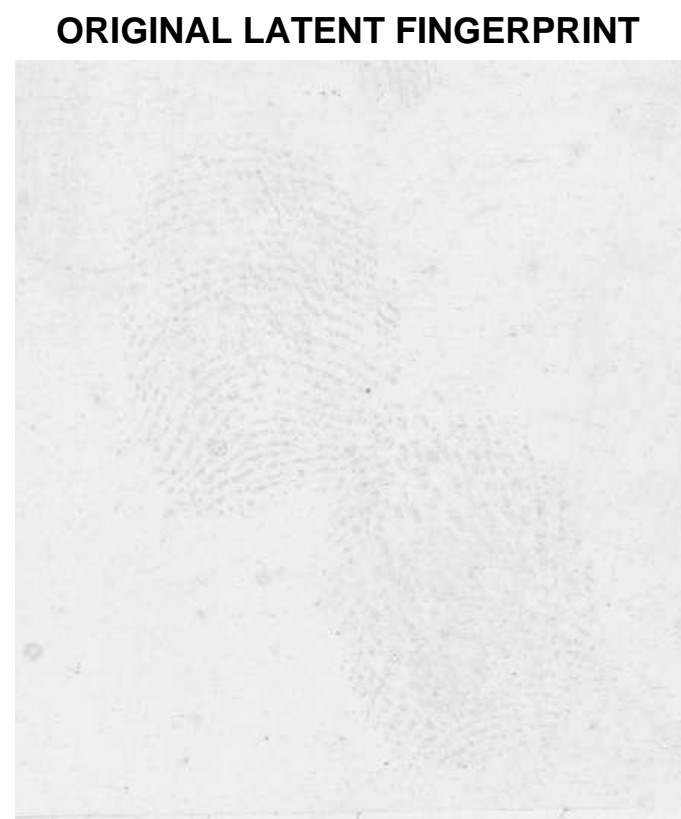

IDL SOBEL FILTERING (x 2)

IDL UNSHARP MASKING (x 5)
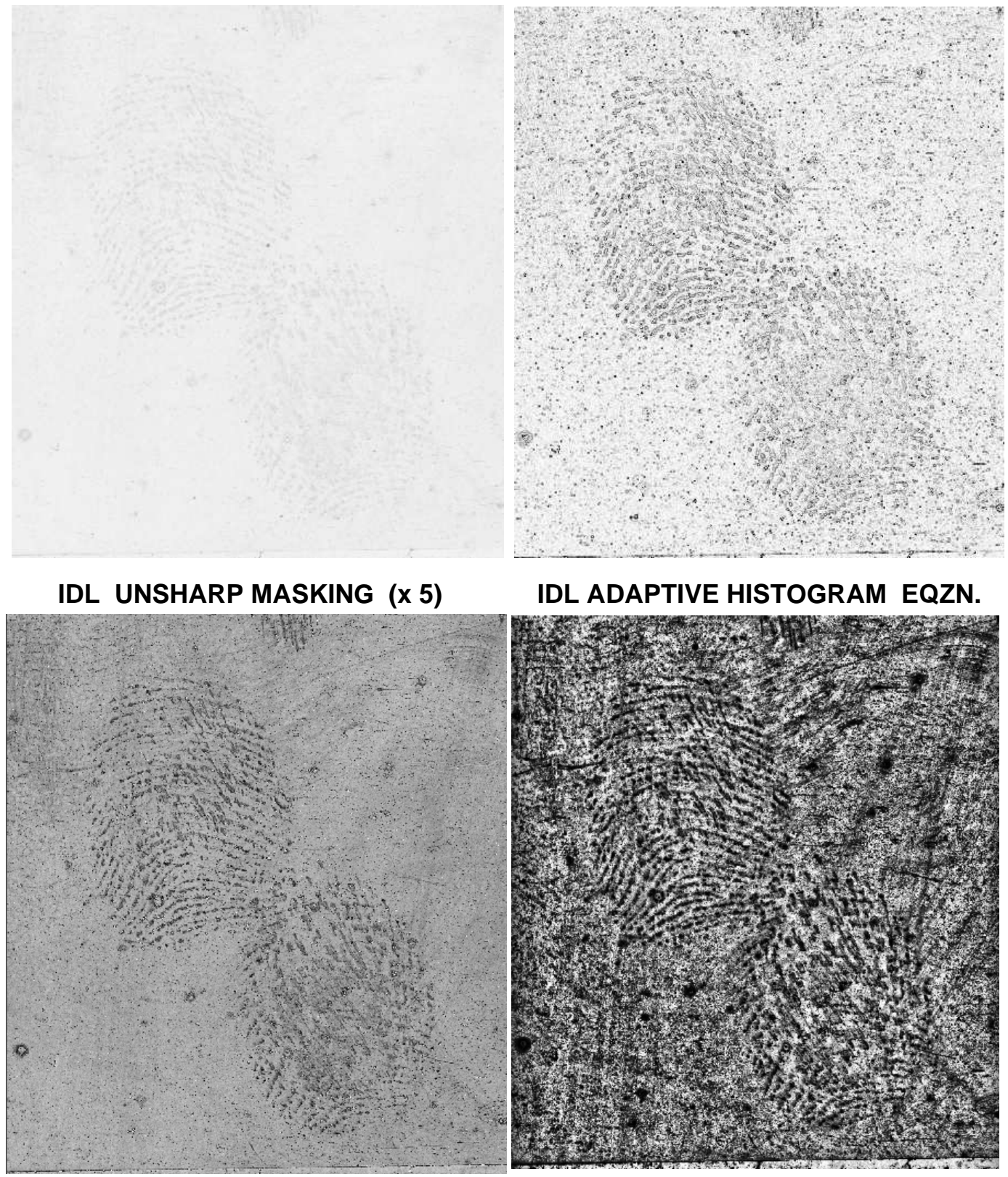

IDL ADAPTIVE HISTOGRAM EQZN.

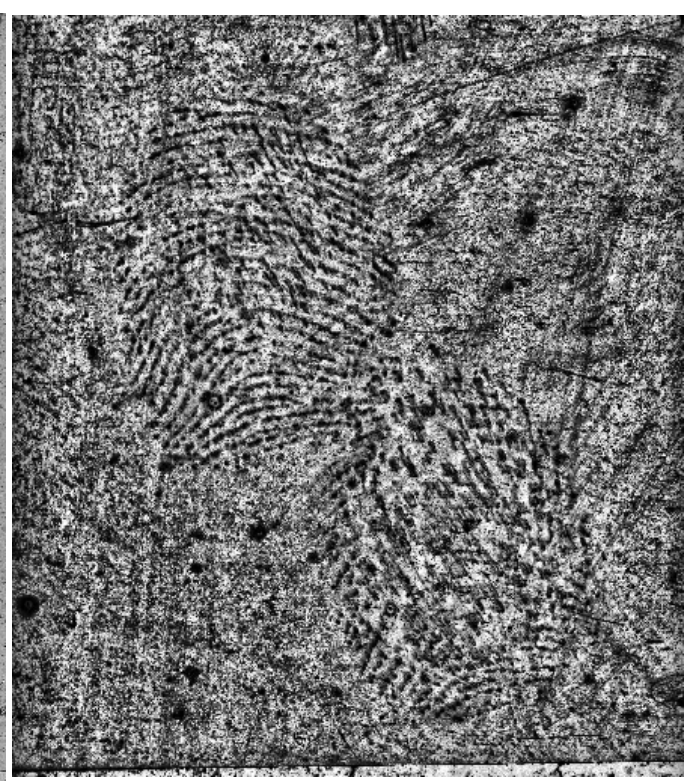

FIG. 12. Reverse images in the additional latent print $(C)$ enhancements shown in Figure 11. 